\title{
Robust Decision Making in a Nonlinear World
}

\author{
Michael R. Dougherty \\ University of Maryland
}

\author{
Rick P. Thomas \\ University of Oklahoma
}

\begin{abstract}
The authors propose a general modeling framework called the general monotone model (GeMM), which allows one to model psychological phenomena that manifest as nonlinear relations in behavior data without the need for making (overly) precise assumptions about functional form. Using both simulated and real data, the authors illustrate that GeMM performs as well as or better than standard statistical approaches (including ordinary least squares, robust, and Bayesian regression) in terms of power and predictive accuracy when the functional relations are strictly linear but outperforms these approaches under conditions in which the functional relations are monotone but nonlinear. Finally, the authors recast their framework within the context of contemporary models of behavioral decision making, including the lens model and the take-the-best heuristic, and use GeMM to highlight several important issues within the judgment and decision-making literature.
\end{abstract}

Keywords: prediction, heuristics, choice, regression, monotone

The nature of the phenomena typically studied in the behavioral and social sciences rarely reflects simple input-output functions; rather, it is often an emergent property of complex social, cognitive, and neural systems. Constructs such as intelligence, anxiety, prejudice, and depression no doubt correspond to some fundamental property of the human condition, yet they also have no tangible corresponding representation in the physical world; they are realized only through psychological measurement. The distinction between phenomena and data is subtle but important. Phenomena are the constructs or entities that theories are meant to explain; data are observables that we use as proxies of the phenomena (Bogen \& Woodward, 1988).

The critical link between data and phenomena is the set of techniques used for modeling the data, as these techniques allow one to state facts about the data that are used to support the theory of the phenomena. Given this critical link, any assumptions made in the course of modeling data have implications for our understanding of the corresponding phenomena. Unfortunately, for the purposes of (statistical) modeling scientists often make strong assumptions about functional relationships when describing data, without realizing (or acknowledging) that assumptions about one's

This article was published Online First February 13, 2012.

Michael R. Dougherty, Department of Psychology, University of Maryland, College Park; Rick P. Thomas, Department of Psychology, University of Oklahoma, Norman.

The research presented in this paper was partially supported by the Center for Advanced Study of Language and Grant SES-0620062 from the National Science Foundation awarded to Michael R. Dougherty. Both authors contributed equally to this paper, and authorship order is arbitrary. We thank Randy Engle and Lael Schooler for providing their data sets and Will Beasley, Gerd Gigerenzer, Paul Hanges, Jorge Mendoza, Louis Narens, J. Brenden Richie, Amber Sprenger, and Erica Yu for their many valuable comments.

Correspondence concerning this article should be addressed to Michael R. Dougherty, Department of Psychology, University of Maryland, College Park, MD 20742. E-mail: mdougher@umd.edu data are not mere abstractions but are statements about the nature of the underlying phenomena to which the data correspond (cf. Berk, 2004).

The link between the statistical modeling of data and the construction of theories about phenomena exists for any theory leveraged against real data. Although it easy to think of the assumptions embodied in statistical models as applying purely at the level of statistical analysis, these assumptions are often (either implicitly or explicitly) carried forth into theory. At the implicit level, the theoretical conclusions drawn from data are conditioned on the assumptions of the statistical analysis upon which the conclusions were based. Violations of assumptions undermine statistical conclusions validity and, by extension, undermine the process of theory testing.

However, the link is even more explicit in cases in which the statistical modeling framework is used as a description of psychological process. Indeed, it is commonplace for statistical models to be exapted as paramorphic models of human information processing (Gigerenzer, 1991). For example, the ANOVA framework was used by Norman H. Anderson's information integration theory (Anderson, 1968, 1970, 1981) and Harold H. Kelley's covariation theory (Kelley, 1967, 1973) to model impression formation and causal attribution. More recently, Bayesian models utilizing principles from statistics and machine learning have been imported into psychology under the guise of computational theories of cognition (Griffiths, Steyvers, \& Tenenbaum, 2007; Griffiths \& Tenenbaum, 2006; Tenenbaum, Griffiths, \& Kemp, 2006). Of particular relevance to the present article is multiple-linear regression, which has had a long and storied history as a descriptive model of human judgment (Brunswik, 1955; Hammond, 1955; Hoffman, 1960; Hogarth \& Karelaia, 2007; Hursch, Hammond, \& Hursch, 1964), a prescriptive model for clinical judgment (Dawes \& Corrigan, 1974; Dawes, Faust, \& Meehl, 1989; Meehl, 1954), and a normative benchmark against which to compare heuristic models (Karelaia \& Hogarth, 2008; Payne, Bettman, \& Johnson, 1993). 
In terms of description, the linear model has been the "workhorse of judgment and decision-making research" (Hogarth \& Karelaia, 2007, p. 734) for over 60 years. It is also intimately tied to the evaluation and modeling of heuristic mechanisms put forth by Gigerenzer and colleagues (Gigerenzer \& Goldstein, 1996; Gigerenzer, Todd, \& the ABC Research Group, 1999). Clearly, the assumptions embodied in the aforementioned statistical models carry forth into the corresponding theoretical frameworks. Thus, assumptions made for descriptive purposes, such as the assumption of linearity, clearly imply that the explanation of the phenomena requires that assumption. However, what if the data, or the underlying phenomena, are not linear?

Our overarching goal in the present paper is to explore the costs of assuming linearity when linearity does not hold and to propose an alternative approach to statistical decision making and behavioral decision making that does not require strong assumptions about one's data. Our approach relaxes assumptions about functional form and therefore allows one to model any relationship (linear or nonlinear) characterized by monotonicity. In what follows, we first review evidence that many psychological phenomena are characterized by nonlinearities. We then describe a general modeling framework, dubbed the general monotone model (GeMM), which allows one to model monotone relations without the need for making precise assumptions about functional form. Using both simulated and real data, we illustrate that GeMM performs as well as or better than standard statistical approaches (including ordinary least squares, robust, and Bayesian regression) in terms of power and predictive accuracy when the nature of data is linear but outperforms these approaches under conditions in which statistical relationships are monotone but nonlinear. Finally, we recast our framework within the context of contemporary models of behavioral decision making, including the lens model and the take-the-best heuristic, and use GeMM to highlight important issues within the judgment and decision-making literature.

\section{Nonlinearities in Psychological Research}

A core assumption of our work is that the functional relationships that describe data within the behavioral and social sciences are often nonlinear. There are many examples of this within psychological science. For instance, many psychophysical judgments are approximated by a logarithmic function (e.g., the Weber-Fechner law). The relationship between practice and skilled performance is approximated by a power function (e.g., the power law of practice, Newell \& Rosenbloom, 1981; but see Heathcote, Brown, \& Mewhort, 2002). Cumulative retrieval functions in verbal-fluency tasks follow a negatively accelerated exponential function (Bousfield \& Sedgwick, 1944). In economics, subjective expected utility is nonlinearly related to value, with the form of the nonlinearity differing for gains, a concave function, and losses, a convex function (Kahneman \& Tversky, 1979). In the decision-making literature, the relationship between perceptions of probability and objective probabilities follows a sigmoidal function (DuCharme, 1970; Erev, Wallsten, \& Budescu, 1994), whereas the relationship between frequency estimates and actual frequency is sometimes exponential (Lichtenstein, Slovic, Fischhoff, Layman, \& Combs, 1978). Finally, perceptions of numbers by children (Thompson \& Opfer, 2008) and adults (Longo \& Lourenco, 2007) are systematically distorted, such that differences between larger values are compressed relative to smaller values, roughly following a logarithmic function. The above examples illustrate the wide variety of data patterns observed in psychological science and therefore serve to ground the central thesis of the work presented here, namely, that the behavioral responses researchers measure are frequently nonlinear. ${ }^{1}$

To be sure, there is good evidence that phenomena within psychological science, or at least our measurement of them, are often nonlinear. Yet, the linear model continues to be one of the most widely used approaches both to the analysis of data and to the construction of theories about phenomena. One important cost of making strong assumptions about the nature of the data is that one risks missing important statistical relationships that may exist but that the linear model is ill equipped to detect. However, a far more important cost is that one could clearly misrepresent the correspondence between the data and the phenomena of interest. As Berk (2004) noted, "Statistical assumptions invoked to justify an analysis of real data are not just statistical abstractions but statements about nature. If the assumptions made so that a statistical analysis plays through are factual nonsense, one surely has to wonder about the conclusions that follow" (p. 2). Put another way, by making rigid assumptions in the modeling of data, one implicitly endorses those assumptions as a description of the underlying phenomena. The conclusions drawn from any statistical analysis are conditional on having satisfied a set of assumptions, and, by extension, so too are any theoretical statements regarding the corresponding phenomena based on those data.

If the functions that best describe behavioral regularities are nonlinear, then the question is how best to model such phenomena. One approach, of course, is to be as precise as possible when making assumptions about data and to fit the function directly, for example, by fitting nonlinear regression models that estimate particular nonlinear functions (e.g., Young, De Leeuw, \& Takane, 1976). This approach is tantamount to endorsing more stringent assumptions about the data. The benefit of this approach is that one may gain a deeper understanding of the phenomena of interest. There are drawbacks, however, as one risks overfitting the data and losing the ability to predict new observations accurately or, worse, mischaracterizing the underlying phenomena (MacCallum, Cornelius, \& Champney, 1979). Moreover, in many cases it is even unreasonable to assume that the behavioral responses observed through psychological measurement necessarily are linearly related to the underlying psychological variable.

Partly because of the problems with modeling via curve fitting, a far more common approach to modeling psychological data involves applying transformations to the data or ignoring the potential for nonlinearities and modeling the data as if it were linear. This approach is evidenced by the widespread use of the linear model in the analysis of data. However, the linear model still suffers from the same problems as does modeling particular func-

\footnotetext{
${ }^{1}$ In some cases the descriptive label afforded by specifying the particular functional form may be useful for theoretical purposes (e.g., identifying laws of practice or psychophysics), but in the vast majority of cases it is unnecessary for descriptive purposes. Indeed, the examples provided above merely represent a sampling of applications in the behavioral sciences where work has been done to identify the function that best approximates the data.
} 
tional relationships; in this case, scientists restrict themselves to the assumption of linearity rather than one or another functional form. Obviously, there may be times in which both the data and the corresponding underlying phenomena are linear, in which case the assumption of linearity is entirely justified both in analyzing the data and in describing the underlying phenomena. However, in a vast majority of cases, researchers do not have privileged access to information beyond the sample at hand that allows them to validate whether particular assumptions, such as linearity, apply at the level of the population or are characteristic of the underlying phenomena. Nevertheless, because the linear model has been shown to be relatively robust to nonlinearities and violations of assumptions, it is often used by default (cf. Cliff, 1996).

Unfortunately, data in the psychological sciences rarely afford one the luxury of making strong inferences about functional form, linear or otherwise. Making matters more difficult is the fact that "few social scientific theories offer any guidance as to function or form whatsoever. Statements like $y$ increases with $X$ (monotonicity)" (Beck \& Jackman, 1998, p. 597) typify the level of specificity offered by most theories (see also Cliff, 1993). Thus, one often cannot fall back on theory as a means of justifying particular assumptions.

In this paper, we advocate a third approach to modeling behavioral data-one that involves making less restrictive assumptions about the data and that is appropriate for modeling any behavioral regularity characterized by monotonicity. The advantage of our proposed approach is that it entails making fewer assumptions, so there is less room for one to violate the assumptions. As we show below, relaxing assumptions about functional form and modeling data at the level of monotonicity can yield better statistical power than models that assume linearity. Moreover, inasmuch as statistical assumptions carry forth into one's theory of the phenomena of interest, statistical algorithms that make fewer assumptions should be preferred as a matter of parsimony. In what follows, we outline one such algorithm that follows this third approach.

\section{The General Monotone Model}

The fundamental goal within GeMM is to find the coefficients (parameter weights) that minimize the lack of fit between the predicted values of $Y$ and the observed values. However, unlike least squares regression procedures, which minimize the squared deviations between the predicted and observed values and solve for coefficients that yield the best linear solution, GeMM minimizes the number of incorrectly predicted paired comparisons and solves for the coefficients that yield the best monotonic (i.e., rank order) solution.

The power to resolve rank order from paired comparisons stems from the fact that the number of constraints on the ordered relationship grows exponentially as a function of $N$, as given by the fact that there are $N(N-1) / 2$ paired comparisons. As pointed out by Shepard $(1962,1966)$ in his development of ordinal multidimensional scaling, these ordinal constraints also enable one to recover close approximations of the metric (i.e., least squares) solutions from data without computing squared distances. Within the context of GeMM, the constraints forced on the procedure by using paired comparisons enable the model to recover close approximations of the metric regression coefficients, without using least squares estimation procedures. The advantage of using paired comparisons as opposed to least squares is that one need not specify the form of the relationship a priori, and there is no need to apply (monotone) transformations to the data to make them fit the assumptions of the model (Cliff, 1993). Moreover, GeMM's estimated coefficients are robust to the presence of outliers and the marginal distribution of the criterion variable, because the rank order ignores the precise distances between points on the criterion. The results of our competitive model tests suggest that GeMM is a stable algorithm that predicts well in a variety of contexts.

The basic framework for implementing GeMM involves three steps:

1. Identify the set of potential models, with each model representing a collection of variables that are to be evaluated or are theoretically meaningful. In the most general case, one might be interested in evaluating all possible subsets (i.e., models) of $k \geq 1$ variables.

2. Estimate the parameter values for each model that maximize the correspondence between the predicted values and the observed values. In GeMM, the goal is to find parameter coefficients that minimize the lack of rankorder correspondence between the predicted and actual values, in terms of the underlying dominance structure.

3. Correct for model complexity. For the purposes here, we have implemented GeMM within the context of the Bayesian information criterion (BIC) framework, though other definitions of model complexity can be readily applied.

In its simplest form, GeMM consists of a one-parameter model (i.e., one predictor), which is used to predict the criterion of interest. In this context, GeMM is actually identical to the wellknown measure of association, tau, specified by Kendall (1938). Thus, to ease the introduction of GeMM, we build the case for GeMM by drawing parallels with Kendall's tau and then extend its application to the multiple predictor case.

To start, assume one wants to know the strength of relationship between two variables, $X$ (e.g., height) and $Y$ (e.g., weight). Tau is a valid measure of the strength of any monotonic relationship, irrespective of the measurement properties of the data, and does not require strong assumptions about functional form (Gonzalez \& Nelson, 1996).

Tau estimates the degree of monotonic relationship by counting the number of paired comparisons for which the ordinal relationship among each pair of $X \mathrm{~s}$ is concordant (i.e., in agreement) or disconcordant (i.e., in disagreement) with its corresponding pair of $Y$ s. For example, in estimating the relationship between height and weight, one need only count the number of pairs of individuals in which the taller person is heavier than the shorter person and the number of pairs of individuals in which the shorter person is heavier than the taller person. If there are 10 individuals in the sample, there are $10(10-1) / 2=45$ such comparisons. Formally, tau is defined as

$$
\begin{aligned}
& \tau(X, Y)=(C-D) / \operatorname{sqrt}\left[\left(\text { Pairs }-T_{\mathrm{p}}\right) *\left(\text { Pairs }-T_{\mathrm{c}}\right)\right] \text { where } \\
& C=\operatorname{Prop}\left(Y_{i}>Y_{j} \cap X_{i}>X_{j}\right)+\operatorname{Prop}\left(Y_{i}<Y_{j} \cap X_{i}<X_{j}\right) \\
& D=\operatorname{Prop}\left(Y_{i}>Y_{j} \cap X_{i}<X_{j}\right)+\operatorname{Prop}\left(Y_{i}<Y_{j} \cap X_{i}>X_{j}\right)
\end{aligned}
$$




$$
\begin{gathered}
T_{\mathrm{p}}=\operatorname{Prop}\left(Y_{i} \geq Y_{j} \cap X_{i}=X_{j}\right)+\operatorname{Prop}\left(Y_{i} \leq Y_{j} \cap X_{i}=X_{j}\right) \\
T_{\mathrm{c}}=\operatorname{Prop}\left(Y_{i}=Y_{j} \cap X_{i} \leq X_{j}\right)+\operatorname{Prop}\left(Y_{i}=Y_{j} \cap X_{i} \geq X_{j}\right) \\
\text { Pairs }=N(N-1) / 2
\end{gathered}
$$

In words, $C$ is the proportion of paired comparisons in which the values of $X$ and $Y$ are in concordance (i.e., the ordinal relationship between $Y_{i}$ and $Y_{j}$ matches the ordinal relationship between $X_{i}$ and $X_{j}$ ). $D$ is the proportion of paired comparisons in which the values of $X$ and $Y$ are in disconcordance (i.e., the ordinal relationship between $Y_{i}$ and $Y_{j}$ is opposite the ordinal relationship between $X_{i}$ and $\left.X_{j}\right) . T_{\mathrm{p}}$ is the proportion of paired comparisons in which the predictor variable is tied, and $T_{\mathrm{c}}$ is the proportion of paired comparisons in which the criterion variable is tied. Pairs is the total number of paired comparisons.

Equation 1 can be rescaled to estimate the Pearson productmoment correlation, $r$. Following Kendall (1970),

$$
r_{\tau} \approx \sin (\pi / 2 \tau)
$$

where $r_{\tau}$ is the value of $r$ estimated from tau. Although Kendall's $\tau$-to- $r$ transformation is not an unbiased estimate of $r$, it is a close approximation even at modest sample sizes $(N=50$; Rupinski \& Dunlap, 1996). Importantly, prior work has shown that the standard error of $r_{\tau}$ is greater than the corresponding standard error of $r$ calculated on the same samples (Rupinski \& Dunlap, 1996), but where the variance of both estimates shrinks with increases in $N$. We return to this issue later.

The advantage of using tau to model statistical relationships is that it is invariant to monotone transformation. Thus, tau is robust, in the sense that within the class of monotone relationships, it is insensitive to the particular functional form. Moreover, the use of Equation 7 to rescale tau provides a means of estimating the value of $r$ under any order preserving (monotone) transformation that linearizes the data, without the need to actually apply the transformation. The advantage of this approach is that it eliminates the need for the researcher to explore the data to find the one transformation that enables the data to meet the assumptions of the statistical test. Moreover, it also removes any subjectivity and, for that matter, implicit bias toward choosing to transform the data or not and, if so, which transformation to choose.

A model-based approach to monotonic association. Much as one can express the linear relationship between $X$ and $Y$ with a least square prediction model, one can express the monotonic relationship between $X$ and $Y$ in terms of an analogous model, as defined by

$$
\hat{Y}=\beta X
$$

In Equation 8, one wishes to find a value for $\beta$ that minimizes the incorrectly predicted paired comparisons. We substitute $\hat{Y}$ for $X$ to reflect this subtle change:

$$
\begin{aligned}
& \tau(\hat{Y}, Y)=(C-D) / \operatorname{sqrt}\left[\left(\text { Pairs }-T_{\mathrm{p}}\right) *\left(\text { Pairs }-T_{\mathrm{c}}\right)\right] \\
& C=\operatorname{Prop}\left(Y_{i}>Y_{j} \cap \hat{Y}_{i}>\hat{Y}_{j}\right)+\operatorname{Prop}\left(Y_{i}<Y_{j} \cap \hat{Y}_{i}<\hat{Y}_{j}\right) \\
& D=\operatorname{Prop}\left(Y_{i}>Y_{j} \cap \hat{Y}_{i}<\hat{Y}_{j}\right)+\operatorname{Prop}\left(Y_{i}<Y_{j} \cap \hat{Y}_{i}>\hat{Y}_{j}\right) \\
& T_{\mathrm{p}}=\operatorname{Prop}\left(Y_{i} \geq Y_{j} \cap \hat{Y}_{i}=\hat{Y}_{j}\right)+\operatorname{Prop}\left(Y_{i} \leq Y_{j} \cap \hat{Y}_{i}=\hat{Y}_{j}\right)
\end{aligned}
$$

$$
T_{\mathrm{c}}=\operatorname{Prop}\left(Y_{i}=Y_{j} \cap \hat{Y}_{i} \leq \hat{Y}_{j}\right)+\operatorname{Prop}\left(Y_{i}=Y_{j} \cap \hat{Y}_{i} \geq \hat{Y}_{j}\right)
$$

With one predictor, only the sign of $\beta$ matters, which reflects the direction of the relationship of $\hat{Y}$ and $Y$. Thus, within the context of simple monotonic prediction (Equation 8), the goal is to assess the degree of monotonic association between $Y$ and its predicted value $(\hat{Y})$. Unlike with predicting metric values, no intercept is necessary because adding a constant to $\hat{Y}$ (in Equation 8) would not affect Equations 9-13.

For convenience, it is easiest to think of the betas for the class of one-parameter models as being constrained to $\{+1,0,-1\}$, such that the relationship between $Y$ and its predicted value $(\hat{Y})$ is always expressed as a positive value of tau, but where the beta coefficient defines the relationship. The simple model in (8) can be generalized to include multiple predictors:

$$
\hat{Y}=\beta_{1} X_{1}+\beta_{2} X_{2}+\ldots+\beta_{k} X_{k}
$$

In this case, the estimated coefficients allow the variables to differentially contribute to the prediction equation to determine the model that maximizes the rank order concurrence between $Y$ and $\hat{Y}$.

Equations 9 through 14 provide the computational basis for computing the degree of monotonic relationship between an additive combination of the predictors (the $X \mathrm{~s}$ ) and a criterion variable $(Y)$. Thus, $1-\tau$ provides the quantity that should be minimized and is at its maximum when prediction is at chance and is zero when prediction is perfect (i.e., $\tau=1$ ).

\section{Generating Weights}

Parameter estimation in GeMM requires searching the parameter space to identify coefficients that maximize the value of tau. Given that the search space grows exponentially with the number of predictors, the parameter space to be searched is extremely large even with a small number of predictors. For example, with $k=9$ predictors, there are $2^{k=9}=512$ possible models, excluding interaction terms, with each parameter requiring estimation in metric space. Fortunately, a number of search algorithms have been developed that make searching complex parameter spaces fairly straightforward (e.g., genetic algorithms, simulated annealing, and memetic algorithms). Thus far, we have used genetic algorithms, which are based on the principles of natural selection and are particularly effective in large and complex search spaces (Goldberg, 2002). Genetic algorithms consist of several steps:

1. Generate a random population of parameter vectors.

2. Evaluate the fitness of each member of the population.

3. Identify those models that are "best" and carry them over to the next population (i.e., elitism).

4. Stochastically sample models (i.e., parents) from the population in the first step, according to their fitness; create a new population of models (i.e., offspring) by directly copying the sampled parents or probabilistically recombining the sampled models; and probabilistically add "noise" to the parameters (i.e., mutation) before adding the offspring to the new population.

5. Return to Step 2. 
This process is implemented iteratively until the algorithm converges on a solution. Genetic algorithms are known to function well in complex search spaces, though they have a tendency to find local minima in some search landscapes. Local minima can be cross-checked by generating new random starting populations through repeated runs.

\section{Occam's Razor: Trading Fit for Complexity}

An important consideration in model building is model complexity: One often wishes to find the best fitting statistical model while minimizing the model complexity (see Myung \& Pitt, 2004). In standard regression contexts, a number of alternatives have been proposed for trading model complexity for fit, including Akaike's information criterion (AIC; Akaike, 1974), Schwarz's information criterion (SIC; Schwarz, 1978; also known as the Bayesian information criterion; BIC), and the risk inflation criterion (RIC; Foster \& George, 1994), among others. All of these alternatives have been developed within least squared or maximum likelihood frameworks, therefore preventing their straightforward application to nonparametric estimators, such as tau. However, the relationship between tau and $r$ stated in Equation 7 enables us to work within the context of the nonparametric estimator tau and still utilize model selection procedures developed for maximum likelihood estimation. In the analyses that follow, we employ a version of the BIC based on $R^{2}$ (see Raftery, 1995), though other model selection procedures can be used. Raftery (1995) showed that the BIC could be estimated from

$$
\mathrm{BIC}=N \log \left(1-R^{2}\right)+k \log (N)
$$

where $N$ is the sample size, $R^{2}$ is the squared multiple correlation, and $k$ is the number of parameters. Substituting Equation 7 for the value of $R^{2}$ yields

$$
\mathrm{BIC}_{\tau}=N \log \left\{1-[\sin (\mathrm{pi} / 2 \tau)]^{2}\right\}+k \log (N) .
$$

Equation 16 is the value of the BIC estimated from the $\tau$-to- $r$ transformation. However, because the value of $r_{\tau}$ shows greater variability than $r$, we use an adjusted form of $r_{\tau}$ based on sample size and the number of predictors used in the regression. In particular, we define $r_{\tau}^{\prime}$ as

$$
r_{\tau}^{\prime}=\sin (\mathrm{pi} / 2 \tau \omega)
$$

where

$$
\omega=(N-P-1) / N \text {. }
$$

$\omega$ is a weighting function based on the number predictors, $P$, used in the regression and sample size, $N$. $\omega$ serves to de-weight the value of tau for smaller sample sizes and therefore reduces the variance of the $\tau$-to-r transformation. Because $\omega$ goes to 1.0 as $N$ increases, the asymptotic value of the $\tau$-to- $r$ transformation is preserved. ${ }^{2}$ Substituting $r_{\tau}^{\prime}$ into Equation 15 gives us

$$
B I C_{\tau}^{\prime}=N \log \left(1-r_{\tau}^{\prime 2}\right)+k \log (N) .
$$

Model selection based on Equation 19 (BIC $\left.{ }_{\tau}^{\prime}\right)$ is assessed on the fit of the model to the data as given by the degree of monotonic relationship expressed by the $\tau$-to- $r$ transformation, adjusted for model complexity. The reliance on $r_{\tau}^{\prime 2}$ as opposed to $r^{2}$ results in a model selection procedure that is invariant to monotone transformation-a property we illustrate next.

\section{Competitive Model Testing: How Does GeMM Compare With the Linear Model?}

Equations 9 through 14 provide the computational basis for GeMM, whereas Equation 19 provides a rule for trading complexity for fit. However, how does GeMM perform relative to the linear model? To address this question we conducted a series of modeling competitions and evaluated GeMM's performance on four criteria: (a) the hit rate (i.e., likelihood of detecting true effects), (b) the false-positive rate (i.e., Type I error rate), (c) the ability to accurately estimate the true population parameter values, and (d) the accuracy of predicting new observations (i.e., cross-validation). Using these four criteria with simulated data, we evaluated three statistical modeling approaches.

1. GeMM with model selection using the $\mathrm{BIC}_{\tau}^{\prime}(\mathrm{GeMM})$. Our implementation of GeMM utilized the above equations and $\mathrm{BIC}_{\tau}^{\prime}$ to determine model fit. Parameter search was conducted using a genetic algorithm. The initial population of weight vectors for the genetic algorithm included $2^{p}$ weight vectors estimated via least squares estimation, where $p$ is the number of predictor variables in the data set. In addition, 2,000 vectors were generated by randomly perturbing the least squared estimated weights, and 4,000 randomly generated weight vectors were also included in the initial population. ${ }^{3}$ The genetic algorithm was run for 10 generations. At each generation the 1,000 vectors with the best fitness value (as determined by the $\mathrm{BIC}_{\tau}^{\prime}$ ) were selected for reproduction, with each new generation constrained to 4,000 new members. In addition, the best 500 models from each generation were copied directly to the sub-

\footnotetext{
${ }^{2}$ Analyses using simulated data indicated that model selection using the BIC without weighting tau by omega led to a slightly liberal selection criterion as manifested by a higher Type I error rate and power (i.e., hit rate) than for the OLS-BIC model selection. Note that our use of omega in Equation 17 is intended to correct for overdispersion in the estimate of $r_{\tau}^{\prime}$. In addition to using the form in Equation 18, we implemented a version in which $\omega=(N-k-1) / N$ and one in which $r_{\tau}$ was adjusted using the formula for adjusted $r^{2}$ as given by $1-\left(1-r_{\tau}^{2}\right)(N-1) /(N-k-1)$. As all of these variations led to approximately the same outcome, we choose to use $(N-P-1) / N$ because it corrects for chance at the level of the experiment, rather than at the level of model selection. We note that a variety of alternative solutions to overdispersion based on the traditional least squares maximum likelihood approach may also be appropriate corrections for overdispersion in GeMM, among them variations of the BIC and the AIC that adjust for variance inflation factor, such as the quasi-AIC (QAIC; Burnham \& Anderson, 1998) and the quasi-BIC (QBIC; Lebreton, Burnham, Clobert, \& Anderson, 1992). However, because these methods involve reliance on least squares methods to compute a variance inflation factor, their use seems inappropriate within the GeMM framework.

${ }^{3}$ The inclusion of the random weight vectors helps prevent GeMM from settling into a local minimum dictated by the OLS solution. Although the inclusion of the least squares regression weights improved the performance of the OLS-BIC model, these least square weights had little impact on the performance of GeMM. Indeed, we implemented two different genetic algorithms for searching the parameter space, and they yielded equivalent patterns
} 
sequent generation. The crossover rate for reproduction was set to .85 , and the mutation rate was set at .02 .

2. Least squares with model selection using the Bayesian information criterion (OLS-BIC). Our implementation of OLS-BIC involved utilizing the genetic algorithm for parameter search and using the BIC as the fitness function. Our implementation of the BIC used $1-R^{2}$ : BIC $=N \log \left(1-R^{2}\right)+k \log (N)$, where $R^{2}$ is the squared multiple correlation, $N$ is the number of observations, and $k$ is the number of parameters in the model.

3. Least squares regression with model selection using the Wald statistic (OLS-Wald). Our implementation of OLS-Wald involved selecting parameters to include in the model based on significance testing using a Wald test with $\alpha=.05$.

The above three approaches were evaluated on data generated from a linear equation with a continuous multivariate distribution and six predictors, three of which were null, and where the other three had coefficients of $.5, .3$, and .2. Thus, the base equation generating the data for the linear environment was

$$
Y=.5 X_{1}+.3 X_{2}+.2 X_{3}+0 X_{4}+0 X_{5}+0 X_{6}+1+e
$$

with $e \sim N(0,1)$. For each simulation run two samples were created: One sample was used to estimate the model parameters, and the second was used for cross-validation. We manipulated sample size across three levels $(N=50,100$, and 250). These analyses were then repeated using a nonlinear environment, which was created by using Equation 20 but then raising $Y$ to the power of $5\left(Y^{5}\right)$. All analyses included 200 simulation runs. The GeMM and OLS-BIC models were conducted with MatLab code available from the authors or online at http://www.bsos.umd.edu/psyc/ dougherty/gemm.html; the OLS-Wald analyses utilized the built-in MatLab multiple-regression function.

\section{Model Comparison Using Simulated Data}

\section{Recovering Latent Data Structures}

Figure 1 plots the performance of GeMM in terms of the hit and false-discovery rates compared to both versions of least squares regression for the linear environment. Two observations should be evident. First, when data conform to strict linearity and multivariate normality, both versions of least squares regression have slightly better statistical power than GeMM. Thus, as should be the case, GeMM is inferior to the linear model when the data are actually linear, though these advantages are rather modest. For example, when $N=100$, GeMM shows equivalent power for detecting the parameter with the strongest relationship $(\beta=.5)$ and nearly equivalent power for the second strongest parameter $(\beta=.3)$. This is remarkable, given that GeMM ignores the metric properties of the data and fits only information contained in the ranks.

Next we investigated GeMM in comparison to OLS under the realistic assumption that data do not typically conform to linearity. Figure 2 plots the results of the simulations for the nonlinear environment. In this case, GeMM shows a substantial advantage over the linear model across all sample sizes. For example, for $N=100$, GeMM shows an improvement of $24 \%$ over both versions of OLS in detecting the second strongest parameter $(\beta=0.3)$; it shows an improvement of $24 \%$ over
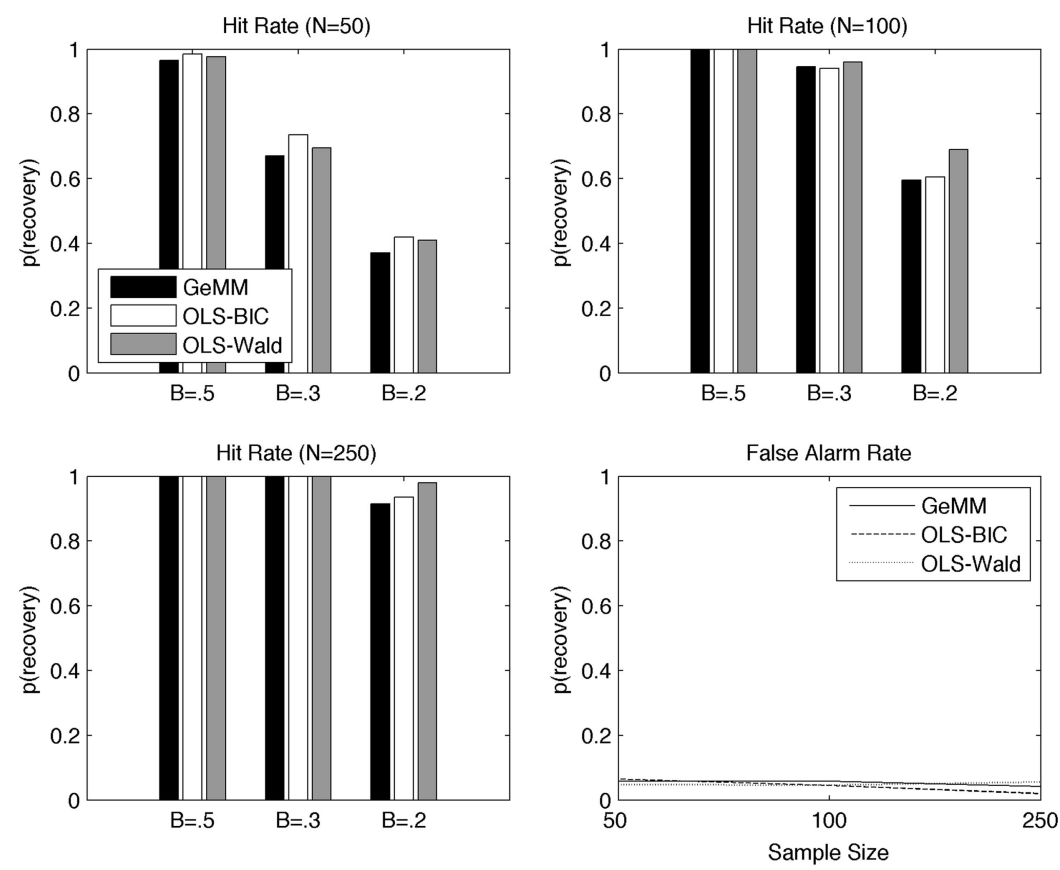

Figure 1. Hit and false alarm rates as a function of sample size for GeMM, OLS-BIC, and OLS-Wald for a linear environment. GeMM = general monotone model; OLS-BIC = least squares with model selection using the Bayesian information criterion; OLS-Wald = least squares regression with model selection using the Wald statistic. 

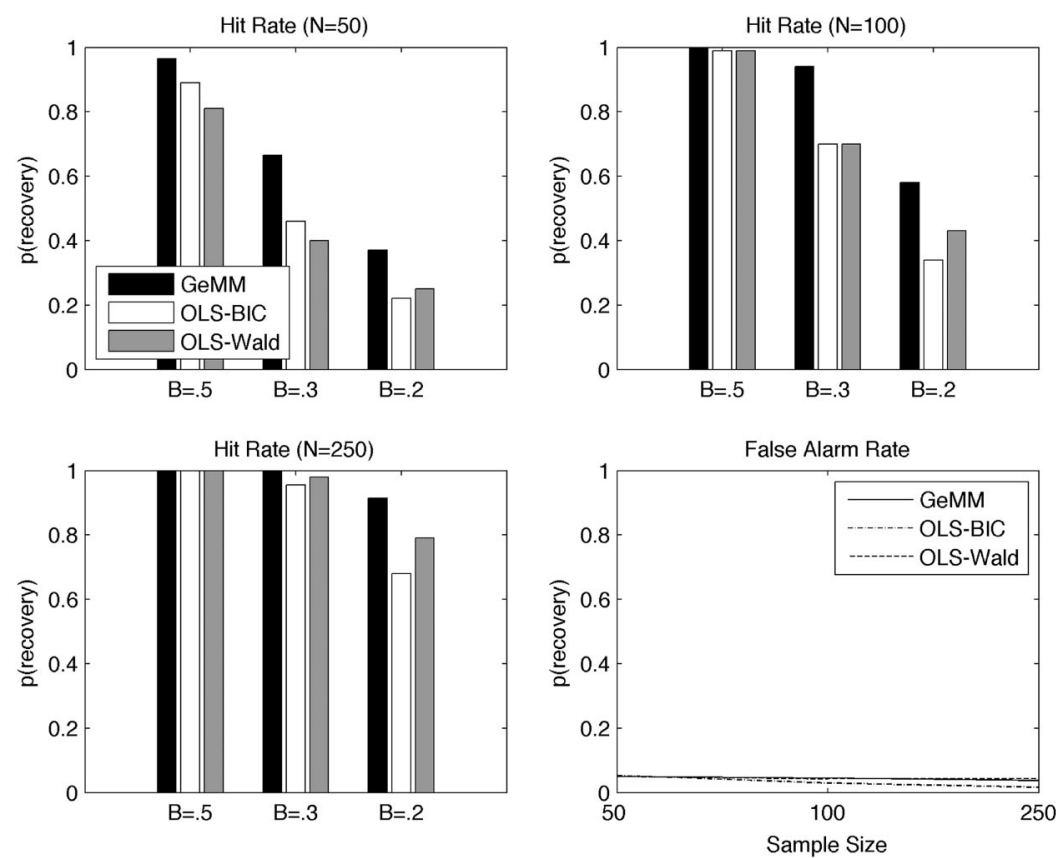

Figure 2. Hit and false alarm rates as a function of sample size for GeMM, OLS-BIC, and OLS-Wald for a nonlinear environment. GeMM = general monotone model; OLS-BIC = least squares with model selection using the Bayesian information criterion; OLS-Wald = least squares regression with model selection using the Wald statistic.

OLS-BIC and a $15 \%$ improvement over OLS-Wald for detecting the weakest parameter $(\beta=0.2)$. Thus, the assumption of linearity made by multiple regression, which is advantageous for the linear environment, is a liability for modeling nonlinear environments. Inasmuch as the nature of data in psychological science is nonlinear, the assumption of linearity will be a liability more often than not. GeMM, on the other hand, is robust to nonlinearity, while showing false discovery rates similar to those for OLS-BIC.

\section{Can GeMM Provide Relatively Accurate Estimates of the Metric Population Parameters?}

Researchers are often concerned with how much a particular variable contributes to the overall predictive accuracy of the model. Therefore, it is useful to know whether GeMM can provide reasonable estimates of the population parameters. In other words, how accurately can GeMM recover the metric parameters that created the observed data?

Figure 3 plots the mean normalized betas estimated by each model. ${ }^{4}$ The population parameters correspond to $.5, .3$, and .2 for the first three parameters and zero for the last three parameters. GeMM's estimates approximate the population parameters. Moreover, GeMM's estimates of these parameters are robust across the environments.

How can GeMM recover the population parameters without capitalizing on the metric properties of the data? The answer to this question stems from the fact that the number of constraints on the rank order increase exponentially with sample size - a property of the method of paired comparisons that Shepard (1962, 1966) argued allowed ordinal multidimensional scaling to approximate metric properties of the data. With reasonable sample sizes, the number of constraints on the ordered relationship is quite high. For example, with $N=100$ and no ties, there are $100(100-1) / 2=$ 4,950 paired comparisons required for determining the best parameters for optimizing the rank order correspondence between $Y$ and $\hat{Y}$. As the number of observations in the rank order increases, the less freedom each value has to vary about its true value. Thus, as $N$ increases, more precise estimates of the betas are required for minimizing rank order inversions. In the limit, these estimates should converge toward the true population parameters. Note, however, that the betas required for minimizing rank order inversions need not correspond exactly to the betas required for minimizing squared error. Therefore, GeMM's estimates are not guaranteed to be unbiased estimates of the population parameters, though inspection of Figure 3 would suggest that they are close approximations.

\section{Predictive Accuracy}

Although Figures 1 and 2 clearly show that GeMM shows statistical power equal to or better than least squares regression techniques, how does it do in predicting new observations? To assess this, we compared GeMM with OLS-BIC and OLS-Wald using cross-validation. The results of these comparisons are presented in Figures 4 and 5, which plot the proportion of concor-

\footnotetext{
${ }^{4}$ The parameter value was set to zero for any parameter not recovered by the model.
} 


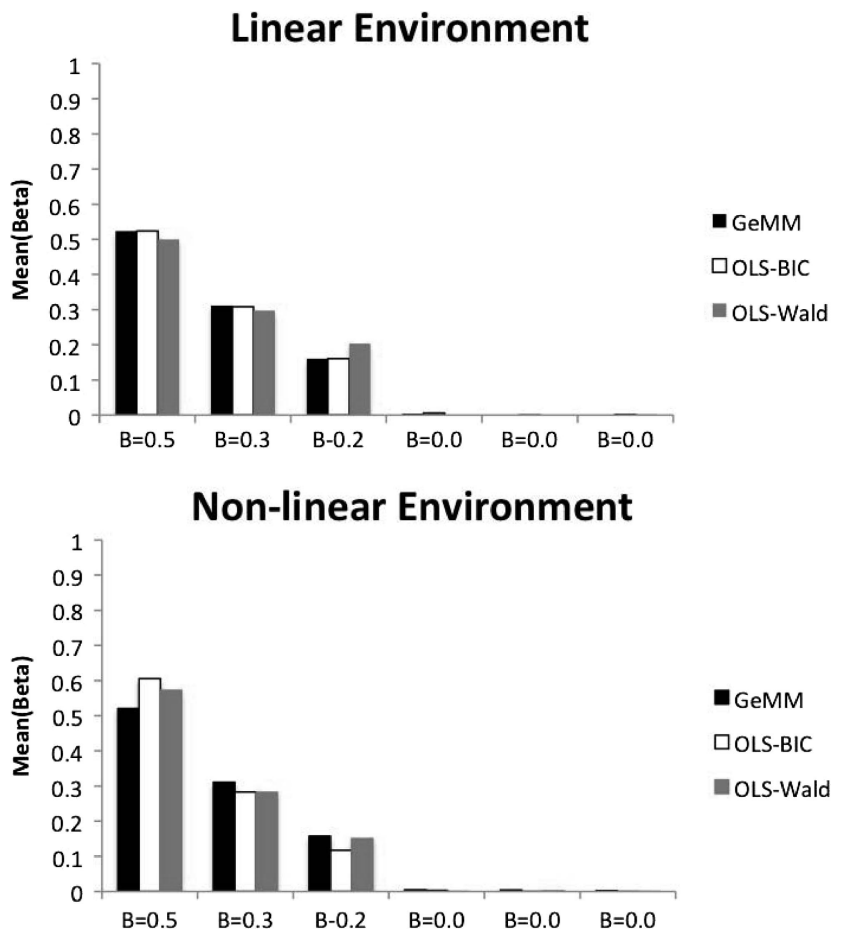

Figure 3. Mean normalized parameter estimates of recovered parameters for GeMM, OLS-BIC, and OLS-Wald in the linear and nonlinear environments for a sample size of 100. GeMM = general monotone model; OLS-BIC $=$ least squares with model selection using the Bayesian information criterion; OLS-Wald = least squares regression with model selection using the Wald statistic.

dances out of all pairwise comparisons, $p(c)=C /[N(N-1) / 2])$, and the estimated tau for a sample size of 100. As can be seen, GeMM shows slightly less predictive accuracy in the crossvalidation sample than do both OLS procedures in the linear environment, but it shows modest improvement in predictive accuracy in the nonlinear environment. The principle reason that GeMM shows better predictive accuracy in the nonlinear case is because it is better at identifying the true model underlying the data. Taken together, these results indicate, that GeMM shows little loss of predictive accuracy in the linear environment, although it is designed to model only monotonicity and shows better predictive accuracy than the linear model for nonlinear environments.

A natural response to our demonstration above is that the linear model does poorly in the nonlinear environment because the appropriate model is indeed nonlinear. We of course agree with this position but add that it is not always clear when data or the corresponding underlying phenomena are nonlinear. Moreover, even if one detects nonlinearities within one's data, it is virtually impossible to know precisely the forms of the nonlinearities and to verify that the nonlinearities are representative of the larger population of scores, as opposed to being idiosyncratic (i.e., random) properties of one's data set. Just as few real data sets truly reflect multivariate normality (Micceri, 1989), it is also likely that few real data sets truly reflect linearity. Modest and perhaps even nonobvious departures from linearity can undermine statistical conclusions.
To illustrate this latter point, we reanalyzed data from Engle, Tuholski, Laughlin, and Conway (1999). Engle et al. were interested in evaluating the factor structure of various measures of cognitive ability. Our use of Engle et al.'s data is somewhat different: We were interested in using their data to evaluate whether GeMM had any practical advantages over various least squares alternatives when applied to data where the true underlying statistical relationship is unknown.

Engle et al. (1999) had 133 participants complete 12 measures of cognitive ability. To reduce computational time, we used only 10 of the 12 available variables. These included two measures of fluid intelligence (Raven's Progressive Matrices, Cattell culture fair test), two standardized achievement tests (verbal SAT and quantitative SAT), three measures of short-term memory span (forward span, backward span, keeping track), and three measures of working memory span (operation span, reading span, and counting span). The use of cognitive ability data for evaluating GeMM was motivated by the assumption that, among real-world measured variables, measures of cognitive ability generally show reasonable distributional properties and approximately satisfy the assumptions of multivariate normality.

As a first step, we analyzed the data to determine whether they met multivariate normality. The results of these analyses were somewhat ambiguous: the Henze-Zirkler test for multivariate normality $(p=.12)$ and the Mardia multivariate test of kurtosis ( $p=$ .09 ) failed to reveal significant departures from multivariate normality, whereas the Mardia test for multivariate skewness was significant $(p<.05) .^{5}$ Thus, we concluded that whatever departures from multivariate normality were present in the data were modest at worst and did not justify transformation.

We examined the statistical power of GeMM versus the two least squares approaches used on the simulated data, plus an additional four approaches (see Appendix), including two versions of robust regression, Bayesian regression with normal priors, and ridge regression. We randomly sampled $50 \%$ of the total sample to form an estimation sample and used the remaining 50\% as the holdout, or cross-validation, sample. For each run, we estimated the model on the estimation sample and then applied the statistical model to predict observations in the cross-validation sample. This was repeated 200 times for each approach. For the sake of comparison, we used the model based on the full sample as the criterion for evaluating statistical power. When we used the full sample, all approaches identified a two-parameter model consisting of quantitative SAT and Cattell's culture fair test as the best fit model for predicting Raven's Progressive Matrices. However, how did the three techniques compare when the estimation sample was reduced by $50 \%$ ?

The results comparing GeMM to the six alternatives based on least squares are presented in Table 1, which provides the probability of recovering each parameter, and Table 2, which provides the fit and cross-validation statistics. As should be clear from examining Table 1, GeMM shows a substantial power advantage over OLS-BIC but especially over OLS-Wald. The robust and

\footnotetext{
${ }^{5}$ Matlab code for the Henze-Zirkler and Mardia tests was obtained from the matlab file exchange (Henze-Zirkler test, Trujillo-Ortiz, HernandezWalls, Barba-Rojo, \& Cupul-Magana, 2007; Mardia test, Trujillo-Ortiz \& Hernandez-Walls, 2003).
} 

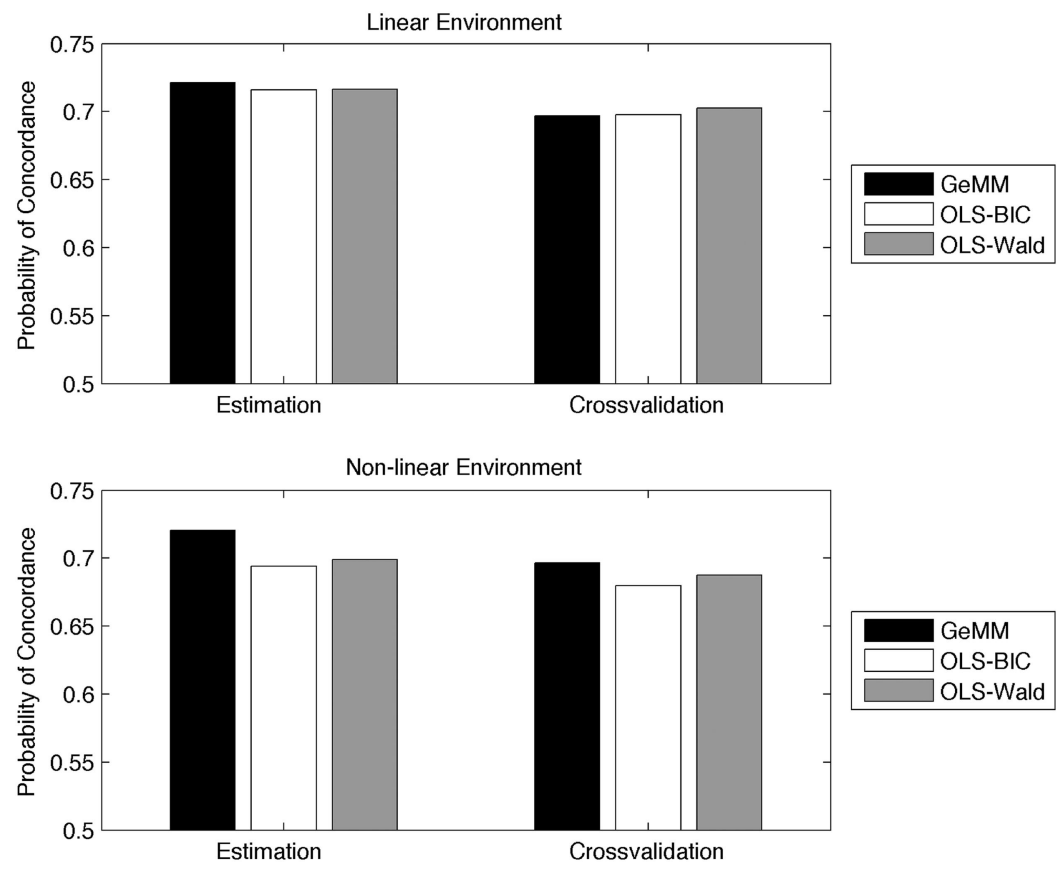

Figure 4. Proportion of concordances for the estimation and cross-validation sample in the linear and nonlinear environments for a sample size of 100. GeMM = general monotone model; OLS-BIC = least squares with model selection using the Bayesian information criterion; OLS-Wald = least squares regression with model selection using the Wald statistic.

Bayesian procedures fared better than OLS-Wald but also underperformed relative to GeMM and OLS-BIC. In fact, GeMM showed roughly a $16 \%$ increase in power over its nearest competitor (OLS-BIC) for detecting quantitative SAT, though it showed a slight decrease in power (roughly $3 \%$ ) for detecting the Cattell culture fair test. Closer inspection of these two variables revealed that quantitative SAT was slightly nonlinearly related to Raven's Progressive Matrices (the best fit quadratic function accounted for nearly $5 \%$ more variance than the linear function when quantitative SAT was regressed onto Raven's), whereas Cattell showed little nonlinearity.

Turning now to predictive accuracy, how well did the various procedures fare in cross validation? These results are presented in Table 2. As can be seen, GeMM outperformed its competitors in terms of monotonic prediction, tau and $P(C)$, while yielding a value for the multiple $r$ that approximated the best fit linear model (OLS-BIC) and which was higher than the multiple $r$ obtained by OLS-Wald, the robust procedures, and Bayes. Thus, although GeMM ignores information captured by the metric fit, it predicts as well or better than the least squared alternatives. The only procedure to outperform GeMM was ridge regression, which is a more complex modeling approach that uses all of the available predictors.

What have we learned from the above analyses? First, it is clear that GeMM is a useful tool for statistical modeling. Obviously, under conditions in which the data are actually linear, least squares regression procedures will be more powerful. However, under the more realistic conditions in which the data are monotone but nonlinear, GeMM is more powerful. Second, GeMM is robust to departures from linearity in a way that standard regression proce- dures are not. Thus, there is no need to test out various transformations on the data to see which one "works" and no need for outlier deletion. The implications of these first two points, however, go well beyond simple lessons for statistical modeling, as they go to the heart of the fundamental goal of science: Making assumptions about the nature of data that do not actually hold, just so a statistical analysis can be performed, can ultimately affect one's theoretical description of the corresponding phenomenon. The evidence taken in support of a theory about phenomena should not rest on questionable assumptions required for the statistical analysis, nor should such evidence be conditional on a particular transformation. Ideally, statements about phenomena should require as few assumptions as necessary. GeMM allows one to model one's data without recourse to data transformation or the default assumption of linearity. A final finding from the initial set of analyses above is that the predictive accuracy of GeMM is as good or better than that of linear regression-a finding that is of particular relevance for evaluating models of behavioral decision making.

\section{GeMM as a Model of Choice}

Having developed GeMM as a tool for describing and analyzing data, we now turn to explicating GeMM within the context of theoretical models of decision making. Of note, we make no claims as to the psychological plausibility of GeMM as a mechanistic-level description of decision behavior. Rather, we view GeMM as serving two complementary purposes. One purpose concerns prescriptive decision making, and the other concerns the description of decision making at the computa- 

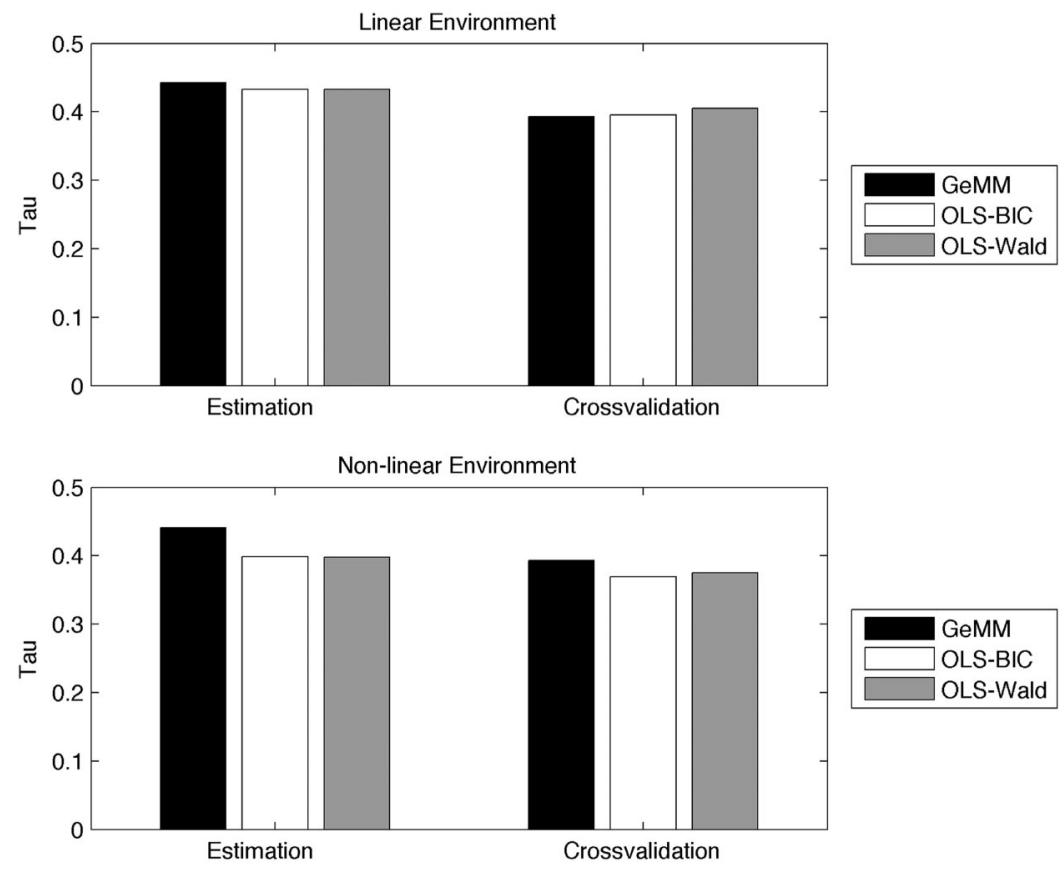

Figure 5. Mean Kendall's tau correlations for the estimation and cross-validation sample in the linear and nonlinear environments for a sample size of 100. GeMM = general monotone model; OLS-BIC = least squares with model selection using the Bayesian information criterion; OLS-Wald = least squares regression with model selection using the Wald statistic.

tional level. When used for prescription, GeMM can be viewed as a model of choice that combines information in a way that respects the monotonic properties of the data when allotted unlimited time and computational resources. The second purpose concerns descriptive modeling and judgment analysis; GeMM allows one to describe cue utilization, in the same way that multiple regression has been used within the context of the lens model (Hammond, Hursch, \& Todd, 1964; Karelaia \& Hogarth, 2008). The use of GeMM in this way has allowed us to identify important theoretical issues that hitherto have been largely neglected in evaluating heuristic models of human judg- ment. Before addressing these issues, however, we first describe a few alternative approaches to modeling decisionmaking behavior.

Egon Brunswik's lens model framework (Brunswik, 1955; Brunswik \& Herma, 1951) is one of the most widespread approaches to modeling judgment and decision making. One of the primary goals of the framework is to understand how people utilize information in inference tasks. Hammond et al. (1964) were among the first to use multiple-linear regression to parameterize and estimate the components of the lens model, and the use of the linear model for estimating parameters in the lens model

Table 1

Probability of Recovering Each Predictor From N/2 Observations

\begin{tabular}{|c|c|c|c|c|c|c|c|c|c|}
\hline Procedure & $\begin{array}{l}\text { Operation } \\
\text { span }\end{array}$ & $\begin{array}{c}\text { Reading } \\
\text { span }\end{array}$ & $\begin{array}{l}\text { Verbal } \\
\text { SAT }\end{array}$ & $\begin{array}{c}\text { Quantitative } \\
\text { SAT }\end{array}$ & $\begin{array}{c}\text { Backward } \\
\text { span }\end{array}$ & $\begin{array}{c}\text { Forward } \\
\text { span }\end{array}$ & $\begin{array}{c}\text { Keeping } \\
\text { track }\end{array}$ & $\begin{array}{c}\text { Counting } \\
\text { span }\end{array}$ & Cattell \\
\hline GeMM & 0.04 & 0.075 & 0.075 & 0.865 & 0.02 & 0 & 0.095 & 0.085 & 0.965 \\
\hline OLS-BIC & 0.05 & 0.035 & 0.03 & 0.71 & 0.06 & 0.005 & 0.135 & 0.095 & 1 \\
\hline OLS-Wald & 0 & 0.05 & 0 & 0.435 & 0.02 & 0.07 & 0.045 & 0.05 & 1 \\
\hline RLS-Wald (Huber) & 0 & 0.05 & 0.005 & 0.575 & 0.005 & 0.045 & 0.02 & 0.045 & 1 \\
\hline RLS-Wald (bi-square) & 0 & 0.07 & 0.01 & 0.585 & 0 & 0.04 & 0.015 & 0.055 & 0.985 \\
\hline BayesianWald & 0 & 0.028 & 0 & 0.389 & 0.011 & 0.072 & 0.038 & 0.022 & 1 \\
\hline Ridge regression & - & - & - & - & - & - & - & - & - \\
\hline
\end{tabular}

Note. The two predictors in bold (Quantitive SAT and Cattell) represent the two predictors that were identified by all of the model selection procedures when using the full data set $(N=133)$. The numbers in each cell represent the probability of recovering each predictor when using only half of the data set $(N / 2=66)$. Note that GeMM has the highest combined recovery probabilities, suggesting that it has power superior to all of the least squared alternatives. The ridge regression model was not used for model selection and therefore used all nine predictors for estimation. GeMM $=$ general monotone model; OLS-BIC = least squares with model selection using the Bayesian information criterion; OLS-Wald = least squares regression with model selection using the Wald statistic; RLS-Wald (Huber) = robust least squares with Huber adjustment; RLS-Wald (bi-square) = robust least squares with bi-square adjustment; Bayesian Wald = Bayesian regression using normal priors with model selection using the Wald statistic; Cattell = Cattell culture fair test. 
Table 2

Fit Indices and Cross-Validation Statistics for All Seven Regression Procedures

\begin{tabular}{|c|c|c|c|c|c|c|}
\hline Procedure & $\mathrm{BIC}_{\tau}^{\prime}$ & $\mathrm{BIC}$ & $P(C)$ & $\tau$ & $r$ & $\bar{k}$ \\
\hline \multicolumn{7}{|l|}{ GeMM } \\
\hline Estimation & \multirow[t]{2}{*}{-32.4973} & \multirow[t]{2}{*}{-40.4052} & 0.7422 & 0.5633 & 0.7255 & \multirow[t]{2}{*}{2.22} \\
\hline Cross-validation & & & 0.7148 & 0.5071 & 0.6989 & \\
\hline \multicolumn{7}{|l|}{ OLS-BIC } \\
\hline Estimation & \multirow[t]{2}{*}{-29.6933} & \multirow[t]{2}{*}{-42.7902} & 0.7312 & 0.5432 & 0.7305 & \multirow[t]{2}{*}{2.12} \\
\hline Cross-validation & & & 0.7122 & 0.5042 & 0.6998 & \\
\hline \multicolumn{7}{|l|}{ OLS-Wald } \\
\hline Estimation & \multirow[t]{2}{*}{-25.7679} & \multirow[t]{2}{*}{-40.9317} & 0.6998 & 0.5108 & 0.7158 & \multirow[t]{2}{*}{1.67} \\
\hline Cross validation & & & 0.6875 & 0.4861 & 0.6778 & \\
\hline \multicolumn{7}{|l|}{ RLS-Wald (Huber) } \\
\hline Estimation & \multirow[t]{2}{*}{-26.9341} & \multirow[t]{2}{*}{-41.4593} & 0.7093 & 0.5214 & 0.7200 & \multirow[t]{2}{*}{1.74} \\
\hline Cross-validation & & & 0.6981 & 0.4988 & 0.6876 & \\
\hline \multicolumn{7}{|c|}{ RLS-Wald (bi-square) } \\
\hline Estimation & \multirow{2}{*}{-27.028} & \multirow{2}{*}{-41.1566} & 0.7094 & 0.5227 & 0.7188 & \multirow[t]{2}{*}{1.76} \\
\hline Cross-validation & & & 0.6985 & 0.5002 & 0.6876 & \\
\hline \multicolumn{7}{|l|}{ Bayesian Wald } \\
\hline Estimation & \multirow[t]{2}{*}{-27.0316} & \multirow[t]{2}{*}{-41.3713} & .6943 & .5060 & .7144 & \multirow[t]{2}{*}{1.56} \\
\hline Cross-validation & & & .6856 & .4875 & .6821 & \\
\hline \multicolumn{7}{|l|}{ Ridge regression } \\
\hline Estimation & \multirow[t]{2}{*}{-1.427} & \multirow[t]{2}{*}{-9.7332} & .7368 & .5472 & .7144 & \multirow[t]{2}{*}{9} \\
\hline Cross-validation & & & .7195 & .5120 & .6973 & \\
\hline
\end{tabular}

Note. $\quad \mathrm{BIC}=$ Bayesian information criterion; $\mathrm{BIC}_{\tau}^{\prime}=$ Bayesian information criterion based on the weighted $\tau$-to- $r$ transformation; $P(C)=$ percent concordance; $\tau=$ Kendall's tau correlation; $r=$ Pearson's $r$ correlation; $k=$ number of parameters in model; GeMM $=$ general monotone model; OLS-BIC $=$ least squares with model selection using the Bayesian information criterion; OLS-Wald = least squares regression with model selection using the Wald statistic; RLS-Wald (Huber) = robust least squares with model selection using the Wald statistic; RLS-Wald (bi-square) = robust least squares with model selection using the Wald statistic; Bayesian Wald = Bayesian regression using normal priors with model selection using the Wald statistic. The ridge regression procedure does not allow for model selection and therefore used all 9 predictors.

subsequently became commonly accepted practice (Hammond et al., 1964; Hartwig \& Bond, 2011; Hastie \& Kameda, 2005; Hursch et al., 1964; Steinmann \& Doherty, 1972; Tucker, 1964; York, Doherty, \& Kamouri, 1987; for a review, see Karelaia \& Hogarth, 2008).

The lens model makes two key assumptions regarding decision behavior. First, it is assumed that the decision maker has some knowledge of the relationship between the criterion of interest $(Y)$ and the cues $\left(x_{i}^{\prime} \mathrm{s}\right)$ as they exist in the environment. Second, judgment about the criterion variable is assumed to be based on an additive combination of some or all of the cues known to the decision maker. Given these first two assumptions, it is easy to see how the linear model can be used to parameterize or estimate which cues a participant utilized in making a set of judgments and how much weight each cue was given. The configuration of cues and their respective weightings are taken as the decision maker's policy. This policy can then be compared to the optimal policy, as given by the parameters estimated on the environment. Clearly, the use of the linear model to characterize a decision maker's policy entails all of the assumptions involved in multiple-linear regression, including that of linearity. Although in the General Discussion we describe the lens model components in detail and reconceptualize them within the GeMM framework, we do not use the lens model in its entirety here. Instead we evaluate its inference engine (i.e., the linear model) in comparison to GeMM on their ability to cross-validate - to predict a holdout sample with cue weights estimated on an estimation sample.
As an alternative model of decision making, Gigerenzer, Hoffrage, and Kleinbö1ting (1991) proposed the probabilistic mental models (PMM) theory for modeling the environmentbehavior interaction. Although this framework builds on the core ideas of Brunswik's (1955) lens model, it does not rely on multiple linear regression. Instead, PMM postulates that people possess a toolbox of heuristic mechanisms that exploit the properties of the environment. One of these heuristic mechanisms is take the best (TTB), which has received a great deal of attention in the literature (see Gigerenzer \& Goldstein, 1996; for a critique, see Dougherty, Franco-Watkins, \& Thomas, 2008). TTB is a three-step algorithm that involves (a) ordering predictor variables according to their predictive accuracy (defined as cue validity, see below), (b) searching the predictor variables from most to least predictive, and (c) terminating search when a cue is found that discriminates between alternatives in a paired comparison.

TTB defines the "best" predictor in terms of cue validity, where cue validity is given by $v_{i}=p\left\{t(a)>t(b) \mid\left[c_{i}(a)=+\right]\right.$ and $\left.\left[c_{i}(b)=-\right]\right\}$. The validity $\left(v_{i}\right)$ of cue $i$, denoted $c_{i}$, on target variables $a$ and $b, t(\mathrm{a})$ and $t(\mathrm{~b})$, is given by the relative frequency with which $t(a)>t(b)$, given that $a$ is positive on cue $i$ and $b$ is negative in reference class $\mathrm{R}$. The terms $t(a)$ and $\mathrm{t}(\mathrm{b})$ correspond to the value of the target variable for case $a$ and $b$, respectively, where the choice set, $\{a, b\}$, is a paired comparison. Note that the formula for cue validity can be rewritten in terms of the number of paired comparisons in which the predictor and criterion are in concordance divided by the number 
of paired comparisons that are either concordances (C) or disconcordances (D): $v=C /(C+D) .{ }^{6}$ It should be clear that the formula for cue validity is an index of monotonic fit, in the same spirit as tau but without penalizing the numerator for disconcordances and ignoring ties in the denominator. Thus, TTB can be viewed as an alternative model to GeMM for capturing monotonicities.

TTB proceeds by evaluating the cue values in the order of their validity. If the cue discriminates, TTB chooses whichever choice alternative has the positive cue value. If the cue does not discriminate, TTB evaluates the next most valid cue and so forth. If none of the cues discriminate, an alternative is chosen at random.

TTB differs from the linear model in two respects. First, TTB uses only a single cue at a time, whereas the linear model integrates across cues. Second, the fit criterion for TTB is based on monotonicity, whereas the fit criterion for the linear model is based on least squares. Thus, any differences in fit when comparing the linear model with TTB could in principle be due to whether or not the cues were integrated, to differences in the fit criteria, or to both. Indeed, our analyses of GeMM in the first half of this paper shows that the use of the linear model in predicting observations in a nonlinear monotone environment leads to suboptimal choice behavior. Of course, it remains to be seen whether GeMM outperforms other algorithms that model monotonicity, such as TTB.

We evaluated the predictive accuracy of GeMM relative to the linear model and TTB within the context of addressing three theoretical issues. The first issue centers on the topic of parsimony. An important construct in evaluating descriptive models of human choice involves the need for such descriptions to respect the cognitive limitations of the human decision maker. Simon (1956) dubbed this bounded rationality, and it has become an increasingly important consideration in the decision-making literature as the focus has turned to describing decision making in terms of heuristic mechanisms. We illustrate that modeling the fit/parsimony trade-off with variable selection criteria such as the BIC can shed new light on the issue of bounded rationality and its relation to models of cue integration, such as the linear model and GeMM.

The second issue addresses the need to minimize incorrect choices and maximize correct choices. We illustrate that models that focus solely on maximizing correct choices, such as TTB, can yield a seemingly paradoxical effect: The number of incorrect choices can increase at a disproportionately greater rate than correct choices. The use of $\mathrm{BIC}_{\tau}^{\prime}$ for model selection can prevent this from happening.

The third issue concerns the very simple proposition that choice models based on a linear least squares fit criterion can be ill equipped for accurately modeling choice behavior. Note that GeMM's goal of maximizing monotonic fit (i.e., tau) is consistent with the goal of maximizing choice but that the parsimony correction ensures that there is a trade-off between choice accuracy and model complexity. Thus, the use of the BIC may come at a cost of choice accuracy, even though the goal of GeMM is to maximize tau. The contrast with the linear model is that linear least squares regression does not directly model choice behavior but rather infers choice accuracy from the least squares fit. We illustrate that the best fit linear model can yield choice accuracy far below that of GeMM, even in cases in which the linear model accounts for a substantially greater proportion of the variance.

\section{Issue 1: A Rational Model of Behavioral Choice Should Trade Fit for Parsimony}

A parsimonious algorithm is one that uses as little information as necessary to do the job. Simplicity is an important element of Simon's bounded rationality and one that we model. Ideally, one wishes to maximize the fit or predictive accuracy of an algorithm, while simultaneously retaining parsimony. The notion of parsimony has been a central construct of the fast and frugal heuristic tradition. Gigerenzer and Goldstein (1996) and others have argued that fast and frugal heuristics require less information upon which to make a decision. Within this tradition, parsimony (i.e., frugality) is defined locally for each paired comparison (i.e., the number of cues one must search in order to discriminate between two objects). We refer to this form of frugality as local parsimony because it applies locally to individual pairs of objects.

Yet, local parsimony (or frugality) as used in the study of fast and frugal heuristics is quite distinct from the notion of parsimony as used in the statistical and computational modeling literatures. Within these literatures, parsimony is defined over the entire class of objects in the judgment set-that is, how many cues are needed to maximize the fit to all of the data (i.e., all paired comparisons). This sort of parsimony is made explicit in model selection procedures such as the BIC, AIC, and RIC and is indexed by $k$ in the BIC. We refer to this form of parsimony as global parsimony, because it applies globally across the entire data set.

Unfortunately, past evaluations of TTB and other fast and frugal heuristics have largely neglected the issue of global parsimony. Although the definitions of local and global parsimony seem to imply different metrics for assessing the parsimony of a model, they are closely related. For example, within the context of GeMM and multiple regression, the local parsimony of the model is always identical to its global parsimony. That is, for each and every paired comparison, GeMM and OLS use an additive combination of $k$ cues. Thus, both the local and global parsimony of GeMM and OLS are equal to $k$. Within the context of TTB, the global parsimony of the model sets the upper bound for local parsimony. That is, TTB may require $k$ cues to make inferences across the entire class of objects (i.e., all possible paired comparisons) but may only use $k_{i}^{*}$ cues on the $i$ th paired comparison, where $k_{i}^{*} \leq k$. Global parsimony, therefore, is given by $\max \left(k_{i}^{*}\right)$, or the maximum number of cues required for any single paired comparison. In most analyses of TTB, researchers report the mean value of $k^{*}$, the average local parsimony, which will often be less than $k$, the global parsimony. The question we address here is, how does TTB perform relative to GeMM and OLS when global parsimony $(k)$ is held constant?

To illustrate the importance of modeling global parsimony, we reanalyzed the data set used in the initial presentation of the TTB heuristic: the cities data set. This data set consisted of all cities in Germany with more than 100,000 inhabitants (i.e., the 83 largest German cities by population), their population value (the criterion), and nine binary predictor cues (Gigerenzer \& Goldstein, 1996). The predictor cues included (a) national capital (Is the city

\footnotetext{
${ }^{6}$ Cue validity rewritten in terms of concordances and disconcordances, $C / C+D$, is similar to the well-known gamma correlation, in which the rank order correlation between two variables is defined as $(C-D) /(C+$ D) (Goodman \& Kruskal, 1979).
} 
the national capital?); (b) exposition site (Was the city once an exposition site?); (c) soccer team (Does the city have a team in the major league?); (d) intercity train (Is the city on the intercity line?); (e) state capital (Is the city a state capital?); (f) license plate (Is the abbreviation only one letter long?); (g) university (Is the city home to a university?); (h) industrial belt (Is the city in the industrial belt?); (i) East Germany (Was the city formerly in East Germany?).

We used a split-half cross-validation procedure to examine the performance of the models using three metrics: the value of $\mathrm{BIC}_{\tau}^{\prime}$, Kendall's tau, and the proportion of concordances. The $\mathrm{BIC}_{\tau}^{\prime}$ for GeMM was calculated as shown above, where $k$ is based on the number of parameters included in the model. The $\mathrm{BIC}_{\tau}^{\prime}$ for TTB was computed by calculating the equivalent of Kendall's tau from the concordances, disconcordances, and ties derived from the TTB algorithm. The value of $k$ used in the $\mathrm{BIC}_{\tau}^{\prime}$ formula was determined by the total number of cues needed to resolve all pairwise comparisons. In this sense, $k$ is actually identical to the $\max k_{i}^{*}$. For the analyses presented below, we varied the value of $k$ parametrically from 1 to $P$, where $P$ was the number of predictors in the data set. For example, when $k=1$, each model was forced to choose the best one-parameter model, when $k=2$, each model was constrained to find the best two-parameter model, and so forth. For each value of $k$, we conducted 100 Monte Carlo runs, with each run consisting of estimation and cross-validation samples. The best fitting parameters for GeMM and OLS were estimated on the estimation sample using the genetic algorithm (restricting the algorithm to including only $k$ non-zero parameters) and then applying this model to the cross-validation sample. For TTB, the estimation sample was used to estimate the cue validity of all $P$ predictors and then to select and order the $k$ best one-parameter models according to cue validity. The cue ordering of the selected $k$ one-parameter models was then applied to the cross validation sample.

Of note, within TTB one cannot directly estimate a Pearson's $r$. However, given that TTB yields all of the components necessary to compute Kendall's tau, it is possible to use the $\tau$-to- $r$ conversion as a proxy for the Pearson's $r$, which allows us to use Equation 19 for $\mathrm{BIC}_{\tau}^{\prime}$, making a straightforward comparison between TTB, GeMM, and OLS.

Model comparison results. Figure 6 plots the mean values for $\mathrm{BIC}_{\tau}^{\prime}$ as a function of the number of predictors included in the model. Two things are of note. First, as illustrated by the $U$-shaped curve, there is a clear trade-off between number of cues and the value of $\mathrm{BIC}_{\tau}^{\prime}$. According to all three algorithms, on average the best model (lowest $\mathrm{BIC}_{\tau}^{\prime}$ ) derived from the estimation sample included two parameters. More generally, across the three algorithms, only models with four or fewer parameters consistently showed negative values for $\mathrm{BIC}_{\tau}^{\prime}$. Thus, even though accuracy continues to improve as more cues are utilized, model selection using the $\mathrm{BIC}_{\tau}^{\prime}$ leads us to prefer models with four or fewer parameters, with the best overall fit shown for models with two parameters. Second, GeMM provides the best overall fit to the estimation sample, as evidenced by lower values of the $\mathrm{BIC}_{\tau}^{\prime}$ (more negative).

We now turn to the assessment of model accuracy. Figure 7 plots the mean percentage of concordances, whereas Figure 8 plots mean values of tau as a function of number of cues for the estimation sample (top panel) and the cross-validation sample (bottom panel). Consistent with the analysis of $\mathrm{BIC}_{\tau}^{\prime}$, GeMM

\section{Cities Data}

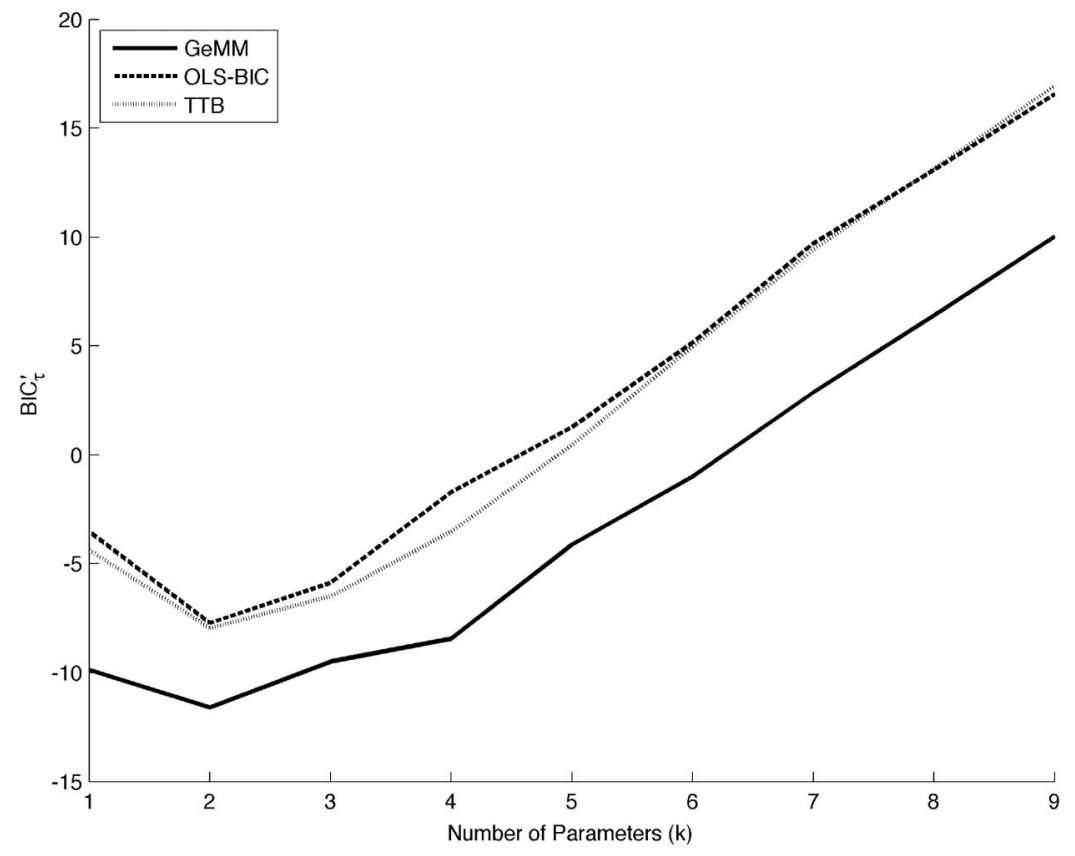

Figure 6. Mean value of $\mathrm{BIC}_{\tau}^{\prime}$ plotted as a function of the number of parameters $(k)$ for each algorithm. GeMM = general monotone model; OLS-BIC = least squares with model selection using the Bayesian information criterion; TTB $=$ take-the-best model. 

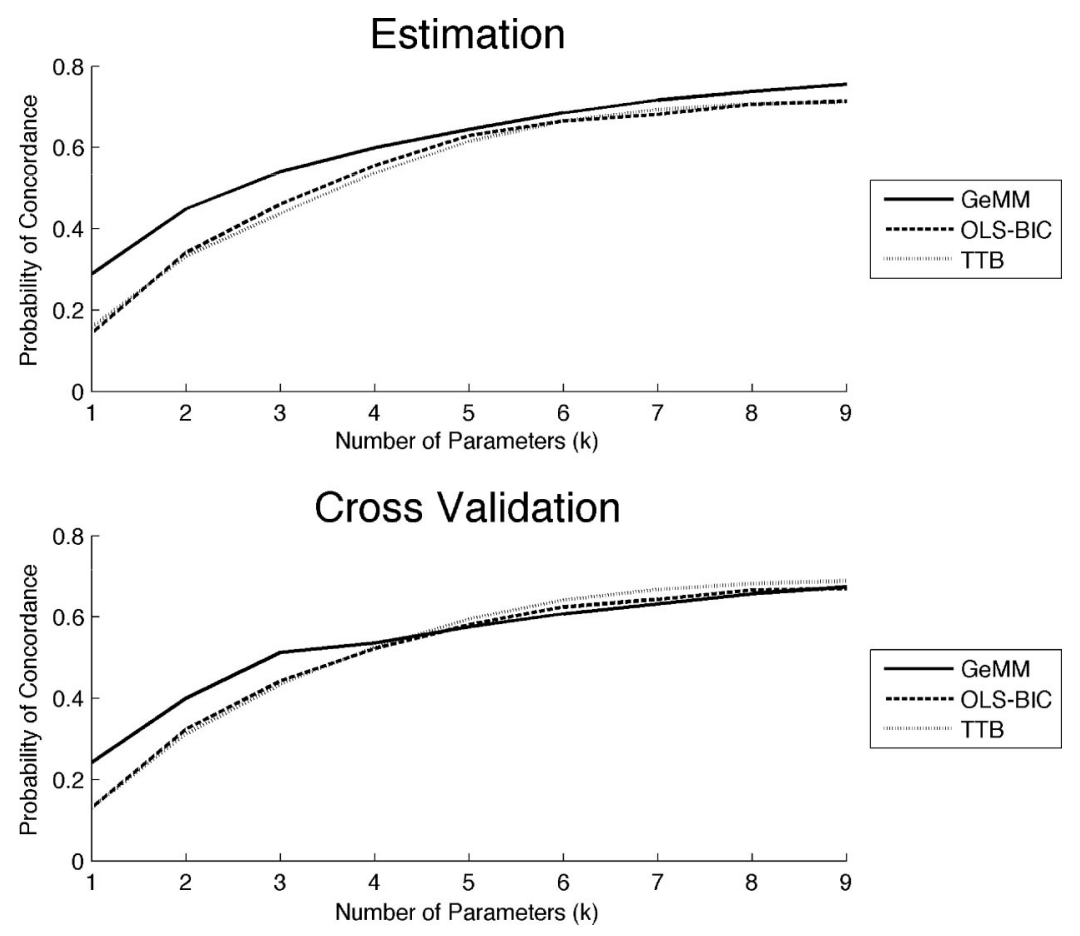

Figure 7. Probability of concordance for the estimation and cross-validation sample for the cities data. $\mathrm{GeMM}=$ general monotone model; OLS-BIC = least squares with model selection using the Bayesian information criterion; TTB $=$ take-the-best model.

outperforms TTB and OLS-BIC on the estimation sample on both measures for all values of $k$. The results for the cross-validation sample illustrate two interesting findings. First, GeMM clearly outperforms both OLS-BIC and TTB for models with fewer parameters. In fact, the predictive accuracy of GeMM (percentage of concordances and tau) exceeds that of TTB and linear regression when the algorithms are restricted to $k<4$, indicating that when disconcordances are factored into the assessment of model fit, GeMM is the clear winner. Note that GeMM is the only model optimized on tau, so it is perhaps unsurprising that it outperforms TTB and OLS on prediction. However, the critical point here is that within least squares regression, one assumes interval level data. Under this assumption, one would expect that maximizing fit at the interval level presupposes that the ordered relationships are well accounted for. As we show below, however, this is not necessarily the case. Moreover, GeMM still does better than both TTB and OLS in terms of percent correct. Thus, after equating the three models for number of parameters and trading fit for complexity, GeMM appears to show a reasonable advantage in predictive accuracy. Interestingly, the advantage for GeMM is not as evident when evaluating tau (see Figure 8).

Second, when allowed to search all nine cues, TTB outperforms both GeMM and OLS-BIC in terms of the proportion of concordances. However, the Kendall's tau for the three-parameter GeMM model $(M=0.463)$ is actually nominally higher than tau for the nine-parameter TTB model $(M=0.461)$, suggesting that a threeparameter GeMM model is nearly as good as a nine-parameter TTB model when evaluated in terms of the monotone correlation. Why does TTB outperform GeMM and OLS on percent concordance but not tau? This is the focus of Issue 2 .

\section{Issue 2: A Rational Model of Behavioral Choice Should Maximize Correction Decisions but Minimize Incorrect Decisions}

Careful inspection of the data in Figures 7 and 8 (cities data) highlights a curious finding: The percent of correct inferences continues to increase even after the value of tau plateaus. How is this possible? The answer lies in the nature of disconcordances, or incorrect choices. Consider the following two equations:

$$
\begin{aligned}
P(C) & =C /\left(C+D+T_{\mathrm{P}}+T_{\mathrm{C}}\right) \\
\tau_{\mathrm{a}} & =(C-D) /\left(C+D+T_{\mathrm{P}}+T_{\mathrm{C}}\right)
\end{aligned}
$$

where $C, D$, and $T$ are defined as concordances, disconcordances, and ties, with ties on the predictor denoted $T_{\mathrm{P}}$ and ties on the criterion denoted $T_{\mathrm{C}}$. $C$ corresponds to a correct choice, whereas $D$ corresponds to an incorrect choice. Equation 21 gives the percent of correct choices, as indexed by the concordances. Equation 22 is a variant of the version of Kendall's tau in Equation 9. For any class of objects, there are $N(N-1) / 2$ paired comparisons. Any paired comparison that is not a $C$ or a $D$ is either a $T_{\mathrm{P}}$ or a $T_{\mathrm{C}}$. As should be clear, the top formula includes no penalty for an incorrect choice, whereas the bottom does. Thus, whether a cue leads to a tie or to an incorrect choice does not affect the value of $P(C)$. To be clear, cues that discriminate between any pair of objects can produce a $C$ or a $D$; for each paired comparison that is resolved as either a $C$ or a $D$, the value of $T_{\mathrm{P}}$ is decreased by one. Thus, theoretically, it is possible for $P(C)$ to increase monotonically even if the preponderance of paired comparisons resolved by a cue are 


\section{Estimation}

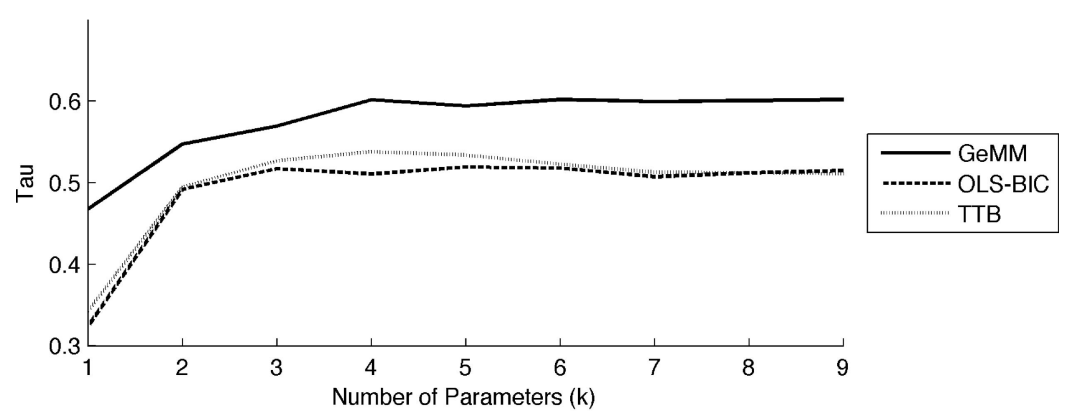

Cross Validation

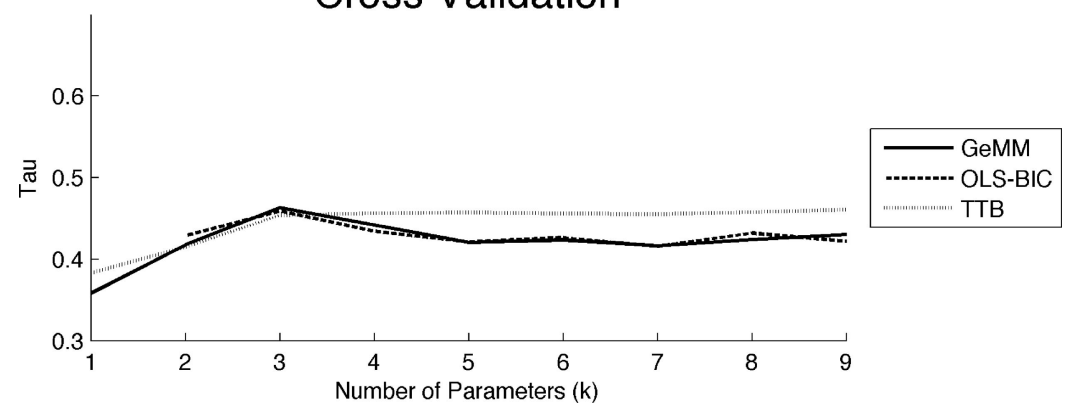

Figure 8. Mean Kendall's tau for the estimation and cross-validation sample for the cities data. GeMM = general monotone model; OLS-BIC = least squares with model selection using the Bayesian information criterion; TTB $=$ take-the-best model

disconcordant. We argue that any rational decision algorithm should be penalized for making incorrect decisions. $P(C)$ therefore provides one with a one-sided assessment of predictive accuracy and can only be interpreted in the context of a metric that takes into consideration incorrect choices, such as tau. In this section, we illustrate that the TTB heuristic ignores the impact of incorrect choices, whereas GeMM does not when the value of $\mathrm{BIC}_{\tau}^{\prime}$ is minimized. As we illustrate below, following a decision strategy that focuses solely on maximizing $P(C)$ can come at a cost of making a disproportionate number of incorrect choices. However, explicitly accounting for incorrect choices in model selection using $\mathrm{BIC}_{\tau}^{\prime}$ can remedy this problem in GeMM and in TTB.

To illustrate, consider an environment in which one wishes to predict $Y$ from two variables, $A$ and $B$. Suppose further that Cue A results in 100 concordances and 25 disconcordances and leaves 200 dyads tied on the predictor. So, with Cue A, $P(C)=100 /(100+25+$ $200)=.307$, and $\tau=(100-25) /(100+25+200)=.231$. Now consider what happens when a second variable is added to the prediction equation that results in 20 additional concordances but 25 additional disconcordances: $P(C)$ increases to $0.369(120 /(125+$ $50+155)$, but tau decreases to $.215([120-50] /[125+50+155])$. In other words, the $6 \%$ increase in correct choices was accompanied by a nearly $8 \%$ increase in incorrect choices. Costs and benefits held equal, it makes little sense for a rational decision maker to use Cue B because it will result in more incorrect choices than correct choices. In this case, ignorance is bliss. Yet, under TTB, Cue B would be used and the disproportionate increase in incorrect choices would be tolerated. In contrast, when using $\mathrm{BIC}_{\tau}^{\prime}$ for model selection within $\mathrm{GeMM}$, any cue added to the model must result in a greater number of concordances relative to disconcordances for it to be justifiably included in the model.

As a real-world demonstration of the example given above, we reanalyzed a data set, previously used to evaluate the accuracy of TTB, that involved predicting professor salaries from a set of five cues, including rank, years in current rank, year degree was earned, sex, and highest degree obtained (see Gigerenzer et al., 1999; Lee $\&$ Cummins, 2004). The data were dichotomized using a median split to allow TTB to use all five cues. Using the full sample $(N=$ 52), we ran GeMM, OLS-BIC, and TTB constraining the algorithms to using $k$ cues, where $k$ was varied from 1 to 5 . The results of these analyses are presented in Figures 9 and 10. Two observations should be evident. First, there is an inverted $U$-shaped function relating tau to the number of parameters for GeMM, OLS-BIC, and TTB, and there is a monotonically increasing function relating proportion of concordances to the number of parameters. This suggests that the inclusion of more parameters in any of the models does not automatically lead to an improvement in choice behavior when incorrect choices are considered. In fact, allowing TTB to search all five cues instead of the four best cues led to an additional 31 correct choices $(C \mathrm{~s})$ but at a cost of 56 incorrect choices $(D \mathrm{~s})$. Second, and perhaps more interesting, the Pearson's $r$ generally showed a monotonic increase as a function of the number of parameters. Thus, if one were to simply maximize the number of concordances or the Pearson's $r$, it would result in a disproportionately large increase in incorrect choices. In contrast, model selection using the $\mathrm{BIC}_{\tau}^{\prime}$ obviates this problem: The best fit model in GeMM is the one that maximizes the trade-off between correct and incorrect (pairwise) choices. 


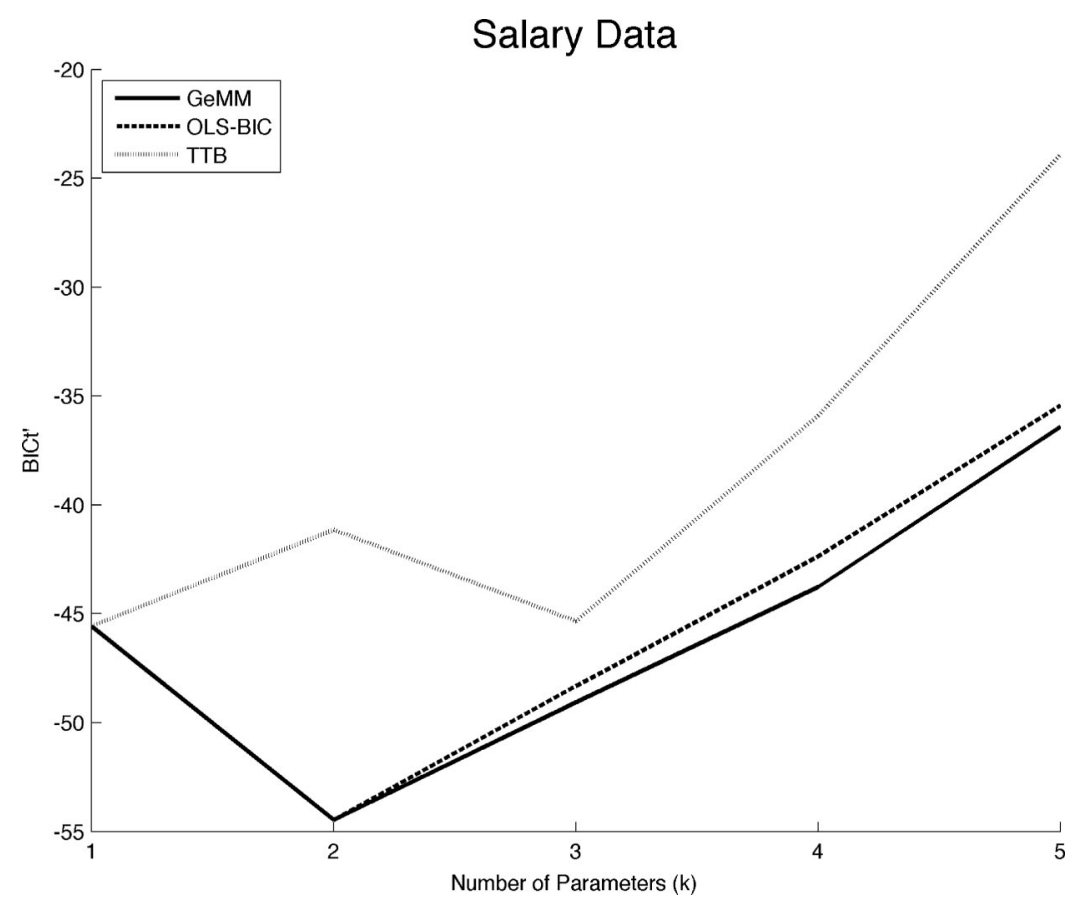

Figure 9. Mean $\mathrm{BIC}_{\tau}^{\prime}$ plotted as a function of the number of parameters $(k)$ for each strategy for the salary data. $\mathrm{GeMM}=$ general monotone model; OLS-BIC $=$ least squares with model selection using the Bayesian information criterion; TTB $=$ take-the-best model.

\section{Issue 3: Decision Models Based on Linear Least Squares Optimization Can Yield Relatively Poor Choice Behavior}

As suggested earlier, the parameters required for minimizing the squared error loss function need not correspond to the parameters needed for minimizing rank order inversions. That is, it is possible for the best fit linear model to account for the most variance, in the traditional sense, and yet do relatively poorly at recovering the rank order solution. Certainly, one should question the validity of any metric solution that fails to recover the more primitive property of ordinality, even if it shows a large $R^{2}$. This point has important implications for the standard applications of Brunswik's lens model that minimizes the squared error loss function.

To illustrate this point, we reanalyzed the cities data set using both GeMM and OLS-BIC. When the full data set was used, the best fit model based on OLS-BIC consisted of three parameters, including the soccer team, exposition site, and national capital cues, and accounted for $84.8 \%$ of the variance, but it yielded a Kendall's tau of 0.517 and a concordance rate of only $38.2 \%$. The best fit GeMM model also consisted of three parameters and included the soccer team, intercity train line, and exposition site cues. In contrast to the best fit linear OLS-BIC model, the best fit GeMM model accounted for only $24.2 \%$ of the variance. However, inspection of the $P(C)$ and tau correlation revealed that GeMM yielded a much higher rank-order correspondence than OLS, with $\tau=0.548$ and a concordance rate of $P(C)=55.4 \%$. Thus, despite the fact that OLS-BIC accounted for over 3 times more variance than GeMM, it performed substantially worse in predicting the rank orders and led to nearly $17 \%(55.4 \%-38.2 \%)$ fewer correct choices. The striking contrast between GeMM and OLS-BIC demonstrates a simple point: The least squares fitting criterion is not designed with the goal of choice maximization. Perhaps even more illuminating is the fact that the best fit OLS-BIC model was actually qualitatively different than the best fit GeMM model, in that they consisted of different predictors.

Although the above findings are of interest for purely statistical reasons, they are also of central importance for the development of prescriptive and descriptive models of choice. The linear model has long been used as a paramorphic model of human decision making and as the normative standard against which human accuracy is compared (Hogarth \& Karelaia, 2007 Karelaia \& Hogarth, 2008). However, it is clearly the case that a decision agent operating under the principle of maximizing choice accuracy may utilize different information than a decision agent operating under the principle of linear least squares.

\section{General Discussion}

We believe that the work presented in this paper illustrates the potential benefits of bringing statistical models in line with the precision of psychological theory and the (nonlinear) nature of the manifest relations in much behavioral data. Using both simulated and real data we demonstrated that GeMM successfully models nonlinear relations without the need to make (overly) precise assumptions about functional form. Moreover, when the functional relation in the data is strictly linear, GeMM suffers negligible losses in terms of power and predictive accuracy. Thus, there seems to be much to gain and little to lose by adopting GeMM as a model of statistical inference. We argued that beyond 


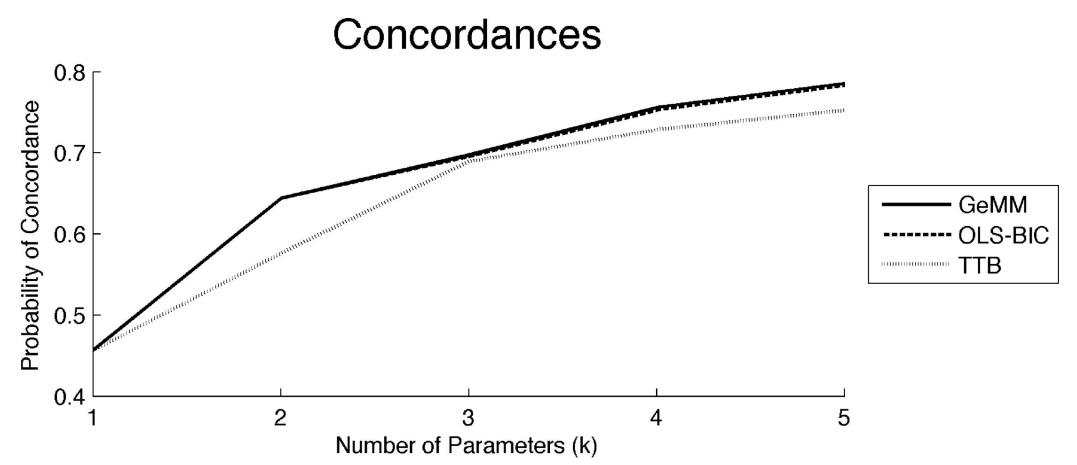

$\mathrm{Tau}$

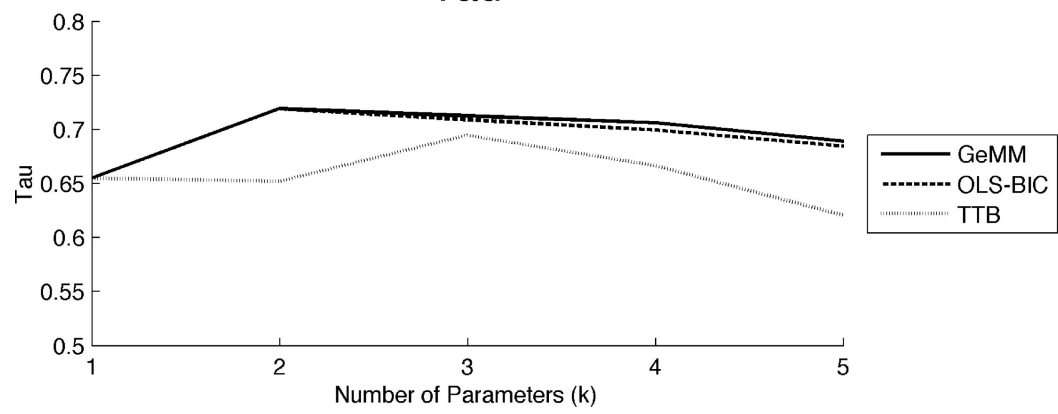

Figure 10. Mean concordances (top panel) and mean taus (bottom panel) plotted as a function of the number of parameters $(k)$ for each strategy for the salary data. GeMM = general monotone model; OLS-BIC = least squares with model selection using the Bayesian information criterion; TTB $=$ take-the-best model.

its use as a statistical model, GeMM could be used as a prescriptive and descriptive framework of judgment and decision making, and then we illustrated this usefulness in identifying several critical issues within behavioral decision theory.

Given the potential for linearities and nonlinearities in one's data, it is instructive to compare the costs and benefits of assuming linearity with the costs and benefits of modeling data under the less restrictive assumption of monotonicity. That is, what does one give up by adopting the less restrictive assumption of monotonicity when the true state of the data is linear? And, what does one give up by adopting the assumption of linearity when the true state of the data is nonlinear? The analyses presented in this paper suggest that the answer to the first question is "very little." As we showed in the first set of analyses, even when data meet the assumptions of linearity, one has little to lose by adopting the less restrictive assumption of monotonicity and modeling data with GeMM. In contrast, the cost of assuming linearity when linearity does not hold can be considerable. Indeed, as we showed using both simulated and real data, applying the linear model to nonlinear data can lead to a substantial reduction in statistical power and poorer predictive accuracy.

One key insight gleaned from our development of GeMM is that it is possible to model any monotonic statistical relationship without making a priori assumptions of functional form. This is made possible by exploiting the transformation on Kendall's tau within a model-based framework. The $\tau$-to- $r$ transformation essentially allows one to model any monotonic nonlinear relationship as though it were linear but without fishing for the transformation that best approximates linearity. This is a major insight, in that it frees the researcher of deciding which, if any, transformation to use on the criterion and eliminates the need to conditionalize statistical conclusion on the choice of a particular transformation (e.g., when two different transformations yield different conclusions). Moreover, because GeMM operates at the level of rank correlation rather than squared deviations, the presence of outliers or extreme scores is of less concern. In our view, using data transformations to reshape the data so that they appear to fit the assumptions of a statistical model is a bit like forcing a round peg into a square hole. It makes more sense to use an algorithm appropriate for the data at hand than to change the data to fit the statistical algorithm (see Cliff, 1996). GeMM is an appropriate approach to statistical modeling of psychological data because it does not require one to adopt overly restrictive assumptions about functional form that may or may not be represented in the data or population.

An important property of GeMM is that the fit criterion is based on the minimization of rank-order inversions. The idea of minimizing rank inversions is consistent with the goal of many applied researchers, who seem less concerned about predicting precise quantitative values than predicting relative values (i.e., rank order; cf. Cliff, 1993, 1996). For example, in personnel selection contexts, it is advantageous to select individuals according to the rank order of applicants, without regard to the quantitative properties of the distribution of scores (Schmidt, 1995; see also Campion et al., 2001). Moreover, even when quantitative values are available, they are often re-expressed in terms of their ordinal or rank properties, as exemplified by the use of percentile ranks when interpreting standardized test scores, such as the WAIS or GRE, and the use of 
linear rank selection in personnel selection decisions (Schmidt, 1995).

Given that the goal of many applied domains is really to identify ordinal relationships, it makes sense to model this property directly, rather than infer it from a least squares solution. In many real-world applications, even a small difference in accurately predicting rank orders can yield large economic or health outcomes. For example, consider health policies designed to identify individuals most at risk for developing breast cancer or policies designed to identify individuals prone to obesity or drug abuse. The difference of $2 \%$ in predicting the rank orders can mean the difference between catching or missing critical high-risk cases. As our analysis of the cities data under Issue 3 illustrates, it is possible for the linear model to substantially underperform GeMM in predicting rank orders, even when the value of $R^{2}$ is substantially higher than that estimated by GeMM. Thus, a high value of $R^{2}$ derived from application of the linear model and assuming interval-level data may give one the illusion that the ordinal properties of the data will follow. However, this need not be the case as the least squared solution is designed to minimize squared error, even if it comes at a cost of mispredicting the ordinal properties of the data. Inasmuch as prediction of ordinal properties is an applied goal, it makes more sense to model the ordinal properties directly than to infer them from the best fit linear model.

Aside from its implications for statistical decision making, our development of GeMM highlighted a number of issues relevant to theories of behavioral decision making. For example, we highlighted the need to control for global model complexity when comparing different decision models. Inasmuch as parsimonious models are preferred, parsimony should be explicitly accounted for when assessing model fit. We illustrated that the predictive accuracy of a popular heuristic model of decision making, take the best (TTB), depends on its global complexity (how many cues it is allowed to search) and that TTB actually does worse than GeMM when global complexity is explicitly modeled. Our conclusion, therefore, is that noncompensatory decision heuristics such as TTB can be advantageous but only when the amount of information (cues) is unconstrained.

A second issue highlighted by our development of GeMM is the need for decision models to explicitly account for incorrect choices as well as correct choices. In past work, the focus of researchers has been on the proportion of correct choices made by TTB. However, the singular focus on correct choices obscures the curious property that more "accurate" models may also commit more incorrect choices. We illustrated that it is possible for the incremental validity of particular cues to be negative, in the sense that using these cues can lead to more incorrect choices than correct choices.

A third issue highlighted by GeMM is that decision models based on linear least squares optimization can appear to perform well when assessed in terms of $R^{2}$ but yield relatively poor choice behavior. This finding illustrates one of the primary limitations of the linear model as a model of choice: It is not designed with the goal of predicting the ordered relations and, therefore, will not tend to maximize (pairwise) choice accuracy. Rather, its goal is to minimize squared error, even if that comes at the cost of choice accuracy.

Although the above findings illustrate the contribution of GeMM to understanding models of decision making, the implica- tions of GeMM for behavioral decision theory go well beyond highlighting these three issues. Indeed, as we illustrate below, GeMM allows for a fundamental reconceptualization of a variety of theoretical frameworks within the decision sciences. We outline of few of these extensions next.

\section{Extensions to Models of Behavioral Choice}

The analyses presented throughout this paper illustrate that GeMM is useful both as a model for statistical inference and as a descriptive model of behavioral choice. However, these analyses have only scratched the surface of its generality. In this section, we outline some natural extensions of GeMM and illustrate its relationship to various models of behavioral choice.

The majority of the work presented throughout the second half of this paper has focused on two seemingly different approaches to judgment and decision making: the lens model, as proposed by Brunswik, and a heuristic-based approach, as advocated most recently by Gigerenzer and colleagues (Gigerenzer \& Goldstein, 1996; Gigerenzer et al., 1999; Goldstein \& Gigerenzer, 2002). Both of these approaches can be reconceptualized within the context of GeMM. Doing so highlights a number of relationships between heuristic algorithms and the lens model approach and also allows us to identify novel variants of the heuristics.

The monotone lens model. One of the most widely used frameworks for modeling decision behavior is the lens model (Hammond et al., 1964; Hartwig \& Bond, 2011; Hastie \& Kameda, 2005; Hursch et al., 1964; Steinmann \& Doherty, 1972; Tucker, 1964; York et al., 1987; for a review, see Karelaia \& Hogarth, 2008). The lens model utilizes multiple linear regression to model both the statistical relationships in the environment and how people use information in inferring properties of the environment (i.e., judgment analysis; for a review, see Karelaia and Hogarth, 2008). Indeed, in a recent review of lens model work, Karelaia and Hogarth (2008) identified over 200 published and unpublished studies using the lens model. This framework is presented in Figure 11, and Table 3 presents interpretations of model constructs. However, we argue that the common use of linear regression in modeling human judgment is misplaced because there are many potential sources of nonlinearities either in the environment (i.e., the true relationships) or in the decision-making process (Einhorn, 1970; Einhorn, Komorita, \& Rosen, 1972; Kim, Yang, \& Kim, 2008).

What are the implications if the state of nature is in fact not strictly linear but conforms to the less special case of monotonicity? In such a case, the linear model is the wrong model and is therefore estimating the wrong weights on the ecology and policy dimensions of the lens model. Thus, deviations in the weights estimated on the environment and the weights estimated on the judge may arise either because the weighting scheme adopted by the judge is ill calibrated with respect to the environment or because the weights identified by the linear model are estimations based on phantom properties of the environment-properties that may not exist! That is, perhaps the linear model is the wrong statistical model against which to compare and model human behavior. Inasmuch as the manifest data are monotonic but not linear, the weights estimated by multiple regression may poorly characterize the judgment policy adopted by the decision maker, the optimal policy suggested by the environment, or both. As 


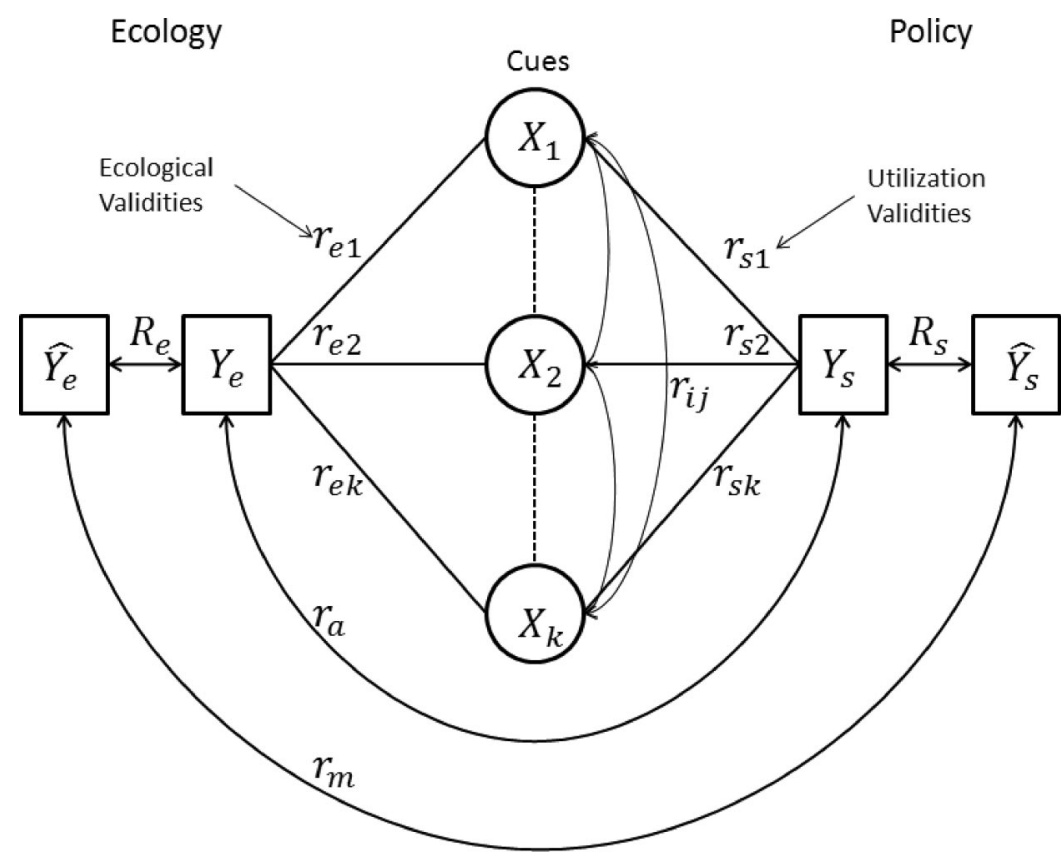

Figure 11. Illustration of the classic lens model framework. The lens model parameters illustrated are described in Table 3.

illustrated under Issue 3, it is even possible for the best fit linear model to be qualitatively different from the best fit monotone model.

The obvious solution to this problem is to adopt a modeling approach that captures monotonic properties but does not assume strict linearity. Thus, we propose GeMM as a paramorphic model of human judgment and decision making. Table 3 recasts the common lens model decomposition in GeMM terms. For the monotone lens model in Table 3 we have chosen to use the Pearson correlation coefficient estimated via Kendall's tau. Although we could just as easily have captured the lens model components with other measures of monotone association, the tau estimated Pearson correlation can be interpreted as the standard lens model with the linear estimate being derived under the less restrictive assumption of monotonicity.

It is also important to point out that the traditional use of the lens model has essentially ignored the issue of model complexity. Our monotone lens model allows for a direct comparison between any model from which paired comparison data can be obtained, including the linear model relying on the $\mathrm{BIC}_{\tau}^{\prime}$. Thus, the fitparsimony trade-off is explicitly considered in terms of the $\mathrm{BIC}_{\tau}^{\prime}$.

In summary, there are many compelling reasons to prefer a monotone lens model. First, linearity is a special case of monotonicity, and, as illustrated by the simulation work, GeMM shows little loss in statistical power when data are linear. The monotonicity assumption also allows for more flexibility and robustness, as illustrated in simulation by GeMM's substantial power advantage as data become increasingly nonlinear but remain monotonic. Finally, when assuming linearity and using least squares optimization, one implicitly endorses a fit metric that tolerates rank inversions in order to minimize squared deviations. This trade-off is not defensible, in our opinion, because rank is a more fundamental measurement property than distance.
Heuristics as monotone decision algorithms. Although GeMM can be considered a monotone version of the lens model, it can also be viewed as a general framework for organizing both compensatory and heuristic models of decision making. Several decision models are closely related to or can be expressed as special cases of GeMM; moreover, our development of GeMM highlights several variations on existing heuristic models and suggests new models.

Table 4 presents several decision models that fit within the GeMM framework: the lens model, Franklin's rule, Dawes' rule, TTB, and minimalist. For example, TTB can be viewed as a variant of GeMM in which one searches through a set of oneparameter models from best to worst until one model discriminates. That is, define a regression model based on GeMM with $P$ parameters (cues). TTB is related to GeMM in that there are $P$ one-parameter models nested within GeMM's $P$-parameter model. TTB differs from GeMM only in the sense that the decision maker is assumed to order the $P$ one-parameter models by validity and search them one at a time. Both Dawes' rule (the equal weights additive rule, or ADD) and Franklin's rule (weighted additive rule, or WADD) can also be considered special cases of GeMM. Both assume that the decision maker chooses between alternatives by combining cues. In the case of Dawes' rule, the cues are combined additively (summed) with equal weight. In the case of Franklin's rule, the cues are weighted by importance before summing (multiple regression is Franklin's rule where the weights are optimized via least squares estimation). Pairwise choice in both models assumes that the decision maker chooses the alternative with the highest sum. WADD and ADD have been investigated extensively as models of choice (Bröder, 2002; Bröder \& Schiffer, 2003; Payne, Bettman, \& Johnson, 1992, 1993). Conceptualized within GeMM, these are merely saturated models that assume that participants combine all 
Table 3

Descriptions of the Lens Model Components in the Original Linear Model and Recast Within GeMM

\begin{tabular}{|c|c|c|c|}
\hline $\begin{array}{l}\text { Linear lens model } \\
\text { component }\end{array}$ & Standard description & $\begin{array}{l}\text { Monotone lens } \\
\text { model component }\end{array}$ & Monotone description \\
\hline$X_{*}$ & Cues are assumed to be metric. & $X_{*}$ & Cues are assumed to be ordinal or metric. \\
\hline$r_{S *}^{*}$ & $\begin{array}{l}\text { Best-fit linear regression coefficients for each cue } \\
\text { regressed on the judge's evaluations. The number of } \\
\text { statistically significant cues is often interpreted as the } \\
\text { frugality or complexity of the judge's policy, and the } \\
\text { magnitude of the linear regression coefficients is } \\
\text { interpreted as the extent to which the cues were } \\
\text { utilized in the judge's policy. }\end{array}$ & $r_{\tau S *}$ & $\begin{array}{l}\text { Best-fit monotone regression coefficients, estimated } \\
\text { via GeMM for each cue regressed on the judge's } \\
\text { evaluations. The number of cues in the best fit } \\
\text { model, using } \mathrm{BIC}_{\tau} \text { to trade fit and complexity, } \\
\text { can be interpreted as the frugality of the judge's } \\
\text { policy, and the magnitude of the monotone } \\
\text { regression coefficients is interpreted as the extent } \\
\text { to which the cues were utilized in the judge's } \\
\text { policy. }\end{array}$ \\
\hline$r_{e *}$ & $\begin{array}{l}\text { Best-fit linear coefficients for each cue regressed on the } \\
\text { criterion. The statistical significance and the } \\
\text { magnitude of the linear coefficients are interpreted as } \\
\text { the validity of the cues to predict the criterion } \\
\text { variable. }\end{array}$ & $r_{\tau e *}$ & $\begin{array}{l}\text { Best-fit monotone regression coefficients, estimated } \\
\text { via GeMM for each cue regressed on the } \\
\text { criterion. The number of cues in the best-fit } \\
\text { model, using } \text { BIC }_{\tau} \text { to trade fit and complexity, } \\
\text { and the magnitude of the monotone regression } \\
\text { coefficients are interpreted as the validity of the } \\
\text { cues to predict the criterion variable. }\end{array}$ \\
\hline$Y_{S}$ & Judgments are assumed to be metric. & $Y_{S}$ & Judgments are assumed to be ordinal or metric. \\
\hline$Y_{e}$ & The criterion variable is assumed to be metric. & $Y_{e}$ & The criterion is assumed to be ordinal or metric. \\
\hline$\hat{Y}_{S}$ & $\begin{array}{l}\text { The predicted values of the best-fit linear model of the } \\
\text { cues regressed on the judge's judgments. }\end{array}$ & $\hat{Y}_{\tau S}$ & $\begin{array}{l}\text { The predicted values of the best-fit GeMM model } \\
\text { of the cues regressed on the judge's judgments. }\end{array}$ \\
\hline$\hat{Y}_{e}$ & $\begin{array}{l}\text { The predicted values of the best-fit linear model of the } \\
\text { cues regressed on the criterion. }\end{array}$ & $\hat{Y}_{\tau e}$ & $\begin{array}{l}\text { The predicted values of the best-fit GeMM model } \\
\text { of the cues regressed on the criterion. }\end{array}$ \\
\hline$R_{S}$ & $\begin{array}{l}\text { Coefficient of determination of the best-fit linear model } \\
\text { of the cues regressed on the judge's judgments. Also, } \\
\text { the Pearson correlation coefficient between the } \\
\text { predicted values of the judge's judgments, estimated } \\
\text { via linear regression, and the observed judgments. } \\
\text { The metric is often interpreted as the ability or fit of } \\
\text { the linear model to capture the judgment process. }\end{array}$ & $R_{\tau S}$ & $\begin{array}{l}\text { The Kendall's tau estimate of the Pearson product } \\
\text { moment correlation coefficient, } r \text {, between the } \\
\text { predicted values of the judge's judgments, } \\
\text { estimated via GeMM regression, and the } \\
\text { observed judgments. The metric can be } \\
\text { interpreted as the ability or fit of the monotone } \\
\text { model to capture the judgment process. }\end{array}$ \\
\hline$R_{e}$ & $\begin{array}{l}\text { Coefficient of determination of the best-fit linear model } \\
\text { of the cues regressed on the criterion. Also, the } \\
\text { Pearson correlation coefficient between the predicted } \\
\text { values of the criterion, estimated via linear } \\
\text { regression, and the observed criterion. The metric is } \\
\text { often interpreted as the ability or fit of the linear } \\
\text { model to capture the ecology. }\end{array}$ & $R_{\tau e}$ & $\begin{array}{l}\text { The Kendall's tau estimate of the Pearson product } \\
\text { moment correlation coefficient, } r \text {, between the } \\
\text { predicted values of the criterion, estimated via } \\
\text { GeMM regression, and the observed criteria. The } \\
\text { metric can be interpreted as the ability or fit of } \\
\text { the monotone model to capture the ecology. }\end{array}$ \\
\hline$r_{a}$ & $\begin{array}{l}\text { The Pearson correlation between the judge's judgments } \\
\text { and the observed criterion. The measure is } \\
\text { interpreted as the quality or accuracy of the judge to } \\
\text { predict the criterion values in terms of variance- } \\
\text { accounted-for. }\end{array}$ & $r_{\tau a}$ & $\begin{array}{l}\text { The Kendall's tau estimate of the Pearson product } \\
\text { moment correlation coefficient, } r \text {, via GeMM } \\
\text { regression, between the criteria and the judge's } \\
\text { judgments. The measure can be interpreted as the } \\
\text { quality or accuracy of the judge to predict the } \\
\text { criterion values. }\end{array}$ \\
\hline$r_{m}$ & $\begin{array}{l}\text { Often referred to as match, the correlation between the } \\
\text { predicted values of the judge's judgments and the } \\
\text { predicted values of the criterion. }\end{array}$ & $r_{\tau m}$ & $\begin{array}{l}\text { The monotone surrogate of match, the Kendall's tau } \\
\text { estimate of the Pearson correlation between the } \\
\text { predicted values of the judge's judgments and the } \\
\text { predicted values of the criterion estimated via } \\
\text { GeMM. }\end{array}$ \\
\hline$r_{i j}$ & $\begin{array}{l}\text { The intercue correlations between the predictors or } \\
\text { cues. }\end{array}$ & $r_{\tau i j}$ & $\begin{array}{l}\text { The Kendall's tau estimate of the Pearson product } \\
\text { moment correlation coefficient, } r \text {, between the } \\
\text { predictors or cues. In process terms, cues that are } \\
\text { highly correlated are assumed to substitute for } \\
\text { each other-a process referred to as vicarious } \\
\text { functioning. }\end{array}$ \\
\hline
\end{tabular}

Note. $\quad$ GeMM $=$ general monotone model.

possible cues (with different weighting schemes) in choosing between alternatives. Note that none of these heuristics explicitly attempt to maximize choice accuracy.

Also represented in Table 4 is the Borda count, which is a voting rule for social preferences in which $N$ choice options are ranked by individuals, where the highest ranked alternative is assigned a ranking of $N$, the second highest ranked alternative is assigned a value of $N-1$, and so forth (Borda, 1784). The winner of a Borda count is the alternative with the highest summed rank. The Borda count is easily expressed as a special case of GeMM, where the predictor variables are viewed as voters, and each voter provides a ranking of all $N$ alternatives. 
Table 4

Decision Models and Relation to GeMM

\begin{tabular}{|c|c|c|c|}
\hline Model & Description & No. parameters $(K)$ & Model selection procedure \\
\hline TTB & $\begin{array}{l}\text { Define all possible 1-parameter models. } \\
\text { Order models according to predictive } \\
\text { validity. For each paired comparison, } \\
\text { search models from best to worst until one } \\
\text { discriminates between the pair of options. }\end{array}$ & $\begin{array}{l}K=\text { number of models required } \\
\text { to discriminate all possible } \\
\text { dyads. }\end{array}$ & $\begin{array}{l}\text { None. Use all possible models } \\
\text { sequentially. }\end{array}$ \\
\hline TTB-select & $\begin{array}{l}\text { Define all possible 1-parameter models. } \\
\text { Order models according to predictive } \\
\text { validity. Select subset of models that } \\
\text { minimize BIC. For each paired } \\
\text { comparison, search selected models from } \\
\text { best to worst until one discriminates } \\
\text { between the pair of options. }\end{array}$ & $\begin{array}{l}K=\text { number of models required } \\
\text { to minimize the } \mathrm{BIC}_{\tau}^{\prime} \text {. }\end{array}$ & $\begin{array}{l}\text { Estimate } \tau \text { based on } \\
\text { concordances and } \\
\text { disconcordances. Transform } \tau \\
\text { to } r \text {, use Equation } 19 \text { or any } \\
\text { other model selection } \\
\text { procedure that trades } \\
\text { parsimony for fit. }\end{array}$ \\
\hline Minimalist & $\begin{array}{l}\text { Define all possible 1-parameter models. For } \\
\text { each paired comparison, randomly choose } \\
\text { model until one discriminates between the } \\
\text { pair of options. }\end{array}$ & $\begin{array}{l}K=\text { number of models required } \\
\text { to discriminate all possible } \\
\text { dyads. }\end{array}$ & None \\
\hline Minimalist-select & $\begin{array}{l}\text { Define all possible 1-parameter models. } \\
\text { Select a subset of models that minimize } \\
\text { BIC. For each paired comparison, } \\
\text { randomly choose a model from the } \\
\text { selected set until one discriminates } \\
\text { between the pair of options. }\end{array}$ & $\begin{array}{l}K=\text { number of models required } \\
\text { to minimize the } \mathrm{BIC}_{\tau}^{\prime} \text {. }\end{array}$ & $\begin{array}{l}\text { Estimate } \tau \text { based on } \\
\text { concordances and } \\
\text { disconcordances. Transform } \tau \\
\text { to } r \text {, use Equation } 19 \text { or any } \\
\text { other model selection } \\
\text { procedure that trades } \\
\text { parsimony for fit. }\end{array}$ \\
\hline $\begin{array}{l}\text { Franklin's rule (weighted } \\
\text { additive model) }\end{array}$ & $\begin{array}{l}\text { Define weight vector. Sum product of weight } \\
\text { vector by data vector. Multiple regression } \\
\text { is the special case where the weight vector } \\
\text { is optimized by minimizing least squares. }\end{array}$ & $\begin{array}{l}K=\text { total number of variables } \\
\text { in the data set. }\end{array}$ & \\
\hline Franklin's rule-select & $\begin{array}{l}\text { Define weight vector. Sum product of weight } \\
\text { vector by data vector. Multiple regression } \\
\text { is the special case where the weight vector } \\
\text { is optimized by minimizing least squares. }\end{array}$ & $\begin{array}{l}K=\text { number of variables } \\
\text { included in the model with } \\
\text { the lowest } \mathrm{BIC}_{\tau}^{\prime} \text {. }\end{array}$ & $\begin{array}{l}\text { Estimate } \tau \text { based on } \\
\text { concordances and } \\
\text { disconcordances. Transform } \tau \\
\text { to } r \text {, use Equation } 19 \text { or any } \\
\text { other model selection } \\
\text { procedure that trades } \\
\text { parsimony for fit. }\end{array}$ \\
\hline $\begin{array}{l}\text { Dawes' rule (unit weight } \\
\text { additive model) }\end{array}$ & $\begin{array}{l}\text { Set weight vector to unit values }(1,0,-1) \text {. } \\
\text { Sum product of weight vector by data } \\
\text { vector. }\end{array}$ & $\begin{array}{l}K=\text { total number of variables } \\
\text { in the data set. }\end{array}$ & $\begin{array}{l}\text { None. Use all predictor variables } \\
\text { simultaneously. }\end{array}$ \\
\hline Dawes' rule-select & $\begin{array}{l}\text { Set weight vectors to unit values. Sum } \\
\text { product of weight vector by data vector. }\end{array}$ & $\begin{array}{l}K=\text { number of variables } \\
\text { included in the model with } \\
\text { the lowest } \mathrm{BIC}_{\tau}^{\prime} \text {. }\end{array}$ & $\begin{array}{l}\text { Estimate } \tau \text { based on } \\
\text { concordances and } \\
\text { disconcordances. Transform } \tau \\
\text { to } r \text {, use Equation } 19 .\end{array}$ \\
\hline Borda count & $\begin{array}{l}\text { Set weight vectors to unit values. Rank } \\
\text { transform the predictors. Sum product of } \\
\text { weight vector by data vector. }\end{array}$ & $\begin{array}{l}K=\text { number of predictors } \\
\text { (voters) in the data set. }\end{array}$ & $\begin{array}{l}\text { None. Use all predictor variables } \\
\text { simultaneously. }\end{array}$ \\
\hline TTB-GeMM & $\begin{array}{l}\text { Define all possible } K \geq 1 \text { parameter models. } \\
\text { Order models according to } \mathrm{BIC}_{\tau}^{\prime} \text {. For each } \\
\text { paired comparison, search models from } \\
\text { best to worst until one discriminates } \\
\text { between the pair of options. }\end{array}$ & - & - \\
\hline Minimalist-GeMM & $\begin{array}{l}\text { Define all possible } K \geq 1 \text { parameter models. } \\
\text { For each paired comparison, randomly } \\
\text { choose model without until one } \\
\text { discriminates between the pair of options. }\end{array}$ & - & - \\
\hline
\end{tabular}

Note. $\quad$ GeMM = general monotone model; $\mathrm{TTB}=$ take the best $\mathrm{BIC}=$ Bayesian information criterion; $\mathrm{BIC}_{\tau}^{\prime}=\mathrm{Bayesian}$ information criterion based on the weighted $\tau$-to- $r$ transformation.

Within GeMM, any data set can be expressed as a Borda count by (a) rank transforming the predictors, (b) assigning unit weights to the predictors such that each "voter" is counted equally, and (c) summing across all predictors. The alternative with the highest summed ranks is the Borda winner. A natural extension of the Borda count suggested by GeMM that is not represented in Table 4 is a weighted Borda count, where each voter is weighted by a validity coefficient derived from a test sample. A reasonable application of the weighted Borda count would involve any prediction task where judges with various levels of expertise or knowledge are predicting the outcome of a contest. Weights estimated from an estimation sample could be taken as an index of each judge's validity (cf. Ho, Hull, \& Srihari, 1994). 
In addition to providing an organizing framework for the above choice rules, GeMM highlights several novel variations of these heuristics. In particular, each of the models described above can be coupled with a model selection procedure that trades fit for parsimony. For example, TTB-select and minimalist-select use the $\mathrm{BIC}_{\tau}^{\prime}$ to reduce complexity. As well, these models can be naturally extended to include any possible parameterization, so that the TTB search algorithm can operate on any ordered set of models containing $k \geq 1$ parameters (TTB-GeMM and minimalistGeMM). For example, under a GeMM formulation, one can order all possible two-parameter models and search them sequentially using TTB's search and stopping rules. Precisely how to define the value for number of parameters $(K)$ and how to select among competing models is yet unclear, so these are left blank in the table. Nevertheless, these are valid decision heuristics that should be explored.

\section{Summary}

The work presented in this paper illustrates the potential benefits of modeling psychological data without recourse to making strong and often untenable assumptions about ones data and without the need for employing data intervention strategies aimed at bringing the data in line with the assumptions of a statistical model. Rather, we argue that a more appropriate approach to data analysis is to utilize a modeling approach that respects the fundamental properties of one's data. GeMM accomplishes just this. GeMM is based on the principle of monotonicity and as such requires much less restrictive assumptions about the modeled relationship. Thus, GeMM permits one to capture the (nonlinear) nature of the manifest relations that exist in much behavioral data and to model the data at a level of precision that is consistent with the precision of most psychological theories.

\section{References}

Akaike, H. (1974). A new look at the statistical model identification. IEEE Transactions on Automatic Control, 19, 716-723. doi:10.1109/ TAC.1974.1100705

Anderson, N. H. (1968). A simple model for information integration. In R. P. Abelson, E. Aronson, W. J. McGuire, T. M. Newcomb, M. J. Rosenberg, \& P. H. Tannenbaum (Eds.), Theories of cognitive consistency: A sourcebook. Chicago, IL: Rand McNally.

Anderson, N. H. (1970). Functional measurement and psychophysical judgment. Psychological Review, 77, 153-170. doi:10.1037/h0029064

Anderson, N. H. (1981). Foundation of information integration theory. New York, NY: Academic Press.

Beck, N., \& Jackman, S. (1998). Beyond linearity by default: Generalized additive models. American Journal of Political Science, 42, 596-627. doi:10.2307/2991772

Berk, R. A. (2004). Regression analysis: A constructive critique. Thousand Oaks, CA: Sage.

Bogen, J., \& Woodward, J. (1988). Saving the phenomena. Philosophical Review, 97, 303-352. doi:10.2307/2185445

Borda, J. C. (1784). "Mémoire sur les élections au scrutin." Histoire de l'Académie Royale des Sciences, Année MDCCLXXI (pp. 657-665). Paris, France: Académie Royale des Sciences.

Bousfield,W. A., \& Sedgwick, C. H. (1944). An analysis of restricted associative responses. Journal of General Psychology, 30, 149-165.

Bröder, A. (2002). Take the best, Dawes' rule, and compensatory decision strategies: A regression based classification method. Quality and Quantity, 36, 219-238. doi:10.1023/A:1016080517126

Bröder, A., \& Schiffer, S. (2003). Take the best versus simultaneous feature matching: Probabilistic inferences from memory and effects of representation format. Journal of Experimental Psychology: General, 132, 277-293. doi:10.1037/0096-3445.132.2.277

Brunswik, E. (1952). The conceptual framework of psychology. Chicago, IL: University of Chicago Press.

Brunswik, E., \& Herma, H. (1951). Probability learning of perceptual cues in the establishment of a weight illusion. Journal of Experimental Psychology, 41, 281-290. doi:10.1037/h0057751

Burnham, K. P., \& Anderson, D. R. (1998). Model selection and inference. New York, NY: Springer-Verlag.

Campion, M. A., Outtz, J. L., Zedeck, S., Schmidt, F. L., Kehoe, J. F., Murphy, K. R., \& Guion, R. M. (2001). The controversy over score banding in personnel selection: Answers to 10 key questions. Personnel Psychology, 54, 149-185. doi:10.1111/j.1744-6570.2001.tb00090.x

Cliff, N. (1993). Dominance statistics: Ordinal analyses to answer ordinal questions. Psychological Bulletin, 114, 494-509. doi:10.1037/00332909.114.3.494

Cliff, N. (1996). Answering ordinal questions with ordinal data using ordinal statistics. Multivariate Behavioral Research, 31, 331-350. doi: 10.1207/s15327906mbr3103_4

Dawes, R. M., \& Corrigan, B. (1974). Linear models in decision making. Psychological Bulletin, 81, 95-106. doi:10.1037/h0037613

Dawes, R. M., Faust, D., \& Meehl, P. E. (1989, March 31). Clinical versus actuarial judgment. Science, 243, 1668-1674. doi:10.1126/science. 2648573

Dougherty, M. R., Franco-Watkins, A. M., \& Thomas, R. (2008). Psychological plausibility of the theory of probabilistic mental models and the fast and frugal heuristics. Psychological Review, 115, 199-213. doi: 10.1037/0033-295X.115.1.199

DuCharme, W. (1970). Response bias explanation of conservative human inference. Journal of Experimental Psychology, 85, 66-74. doi:10.1037/ h0029546

Einhorn, H. J. (1970). The use of nonlinear, noncompensatory models in decision making. Psychological Bulletin, 73, 221-230. doi:10.1037/ h0028695

Einhorn, H. J., Komorita, S. S., \& Rosen, B. (1972). Multidimensional models for the evaluation of political candidates. Journal of Experimental Social Psychology, 8, 58-73. doi:10.1016/0022-1031(72)90061-3

Engle, R. W., Tuholski, S. W., Laughlin, J. E., \& Conway, A. R. A. (1999). Working memory, short-term memory and general fluid intelligence: A latent variable approach. Journal of Experimental Psychology: General, 128, 309-331. doi:10.1037/0096-3445.128.3.309

Erev, I., Wallsten, T. S., \& Budescu, D. V. (1994). Simultaneous over- and underconfidence: The role of error in judgment processes. Psychological Review, 101, 519-527. doi:10.1037/0033-295X.101.3.519

Foster, D. P., \& George, E. I. (1994). The risk inflation criterion for multiple regression. Annals of Statistics, 22, 1947-1975. doi:10.1214/ aos/1176325766

Gigerenzer, G. (1991). From tools to theories: A heuristic of discovery in cognitive psychology. Psychological Review, 98, 254-267. doi:10.1037/ 0033-295X.98.2.254

Gigerenzer, G., \& Goldstein, D. (1996). Reasoning the fast and frugal way: Models of bounded rationality. Psychological Review, 103, 650-669. doi:10.1037/0033-295X.103.4.650

Gigerenzer, G., Hoffrage, U., \& Kleinbö1ting, H. (1991). Probabilistic mental models: A Brunswikian theory of confidence. Psychological Review, 98, 506-528. doi:10.1037/0033-295X.98.4.506

Gigerenzer, G., Todd, P. M., \& the ABC Research Group. (1999). Simple heuristics that make us smart. New York, NY: Oxford University Press. Goldberg, D. E. (2002). The design of innovation: Lessons from and for competent genetic algorithms. Reading, MA: Addison-Wesley. 
Goldstein, D. G., \& Gigerenzer, G. (2002). Models of ecological rationality: The recognition heuristic. Psychological Review, 109, 75-90. doi: 10.1037/0033-295X.109.1.75

Gonzalez, R., \& Nelson, T. O. (1996). Measuring ordinal association in situations that contain tied scores. Psychological Bulletin, 119, 159-165. doi:10.1037/0033-2909.119.1.159

Goodman, L. A., \& Kruskal, W. H. (1979). Measures of association for cross classifications. New York, NY: Springer-Verlag.

Griffiths, T. L., Steyvers, M., \& Tenenbaum, J. B. (2007). Topics in semantic representation. Psychological Review, 114, 211-244. doi: 10.1037/0033-295X.114.2.211

Griffiths, T. L., \& Tenenbaum, J. B. (2006). Optimal predictions in everyday cognition. Psychological Science, 17, 767-773. doi:10.1111/ j.1467-9280.2006.01780.x

Hammond, K. R. (1980). Introduction to Brunswikian theory and methods. In K. R. Hammond \& N. E. Wascoe (Eds.), Realizations of Brunswik's experimental design (pp. 1-11). San Francisco, CA: Jossey-Bass.

Hammond, K. R., Hursch, C. J., \& Todd, F. J. (1964). Analyzing the components of clinical inference. Psychological Review, 71, 438-456. doi: $10.1037 / \mathrm{h} 0040736$

Hartwig, M., \& Bond, C. R. (2011). Why do lie-catchers fail? A lens model meta-analysis of human lie judgments. Psychological Bulletin, 137, 643-659. doi:10.1037/a0023589

Hastie, R., \& Kameda, T. (2005). The robust beauty of majority rules in group decisions. Psychological Review, 112, 494-508. doi:10.1037/ 0033-295X.112.2.494

Heathcote, A., Brown, S., \& Mewhort, D. J. K. (2002). Quantile maximum likelihood estimation of response time distributions. Psychonomic Bulletin \& Review, 9, 394-401. doi:10.3758/BF03196299

Ho, T. K., Hull, J. J., \& Srihari, S. N. (1994). Decision combination in multiple classifier systems. IEEE Transactions on Pattern Analysis and Machine Intelligence, 16, 1, 66-75. doi:10.1109/34.273716

Hoffman, P. J. (1960). The paramorphic representation of clinical judgment. Psychological Bulletin, 57, 116-131. doi:10.1037/h0047807

Hogarth, R. M., \& Karelaia, N. (2007). Heuristic and linear models of judgment: Matching rules and environments. Psychological Review, 114, 733-758. doi:10.1037/0033-295X.114.3.733

Hursch, C. J., Hammond, K. R., \& Hursch, J. L. (1964). Some methodological considerations in multiple-probability studies. Psychological Review, 71, 42-60. doi:10.1037/h0041729

Kahneman, D., \& Tversky, A. (1979). Prospect theory: An analysis of decision under risk. Econometrica, 47, 263-291. doi:10.2307/1914185

Karelaia, N., \& Hogarth, R. M. (2008). Determinants of linear judgment: A meta-analysis of lens model studies. Psychological Bulletin, 134, 404-426. doi:10.1037/0033-2909.134.3.404

Kelley, H. H. (1967). Attribution theory in social psychology. In D. Levine (Ed.), Nebraska symposium on motivation: Vol. 15 (pp. 192-238). Lincoln: University of Nebraska Press.

Kelley, H. H. (1973). The process of causal attribution. American Psychologist, 28, 107-128. doi:10.1037/h0034225

Kendall, M. (1938). A new measure of rank correlation. Biometrika, 30, 81-89.

Kendall, M. G. (1970). Rank correlation methods (4th ed). London, England: Griffin.

Kim, C., Yang, K., \& Kim, J. (2008). Human decision-making behavior and modeling effects. Decision Support Systems, 45, 517-527. doi: 10.1016/j.dss.2007.06.011

Lebreton, J., Burnham, K. P., Clobert, J., \& Anderson, D. R. (1992). Modeling survival and testing biological hypotheses using marked animals: A unified approach with case studies. Ecological Monographs, 62, 67-118. doi:10.2307/2937171

Lee, M. D., \& Cummins, T. D. R. (2004). Evidence accumulation in decision making: Unifying the "take the best" and the "rational" models.
Psychonomic Bulletin \& Review, 11, 343-352. doi:10.3758/ BF03196581

Lichtenstein, S., Slovic, P., Fischhoff, B., Layman, M., \& Combs, B. (1978). Judged frequency of lethal events. Journal of Experimental Psychology: Human Learning and Memory, 4, 551-578.

Longo, M. R., \& Lourenco, S. F. (2007). Spatial attention and the mental number line: Evidence for characteristic biases and compression. $\mathrm{Neu}$ ropsychologia, 45, 1400-1407. doi:10.1016/j.neuropsychologia 2006.11.002

MacCallum, R. C., Cornelius, E. T., \& Champney, T. (1979). Validity and cross-validity of metric and nonmetric multiple regression. Applied Psychological Measurement, 3, 463-468. doi:10.1177/014662167900300404

Meehl, P. E. (1954). Clinical vs. statistical prediction: A theoretical analysis and a review of the evidence. Minneapolis: University of Minnesota Press.

Micceri, T. (1989). The unicorn, the normal curve, and other improbable creatures. Psychological Bulletin, 105, 156-166. doi:10.1037/00332909.105.1.156

Myung, J. I., \& Pitt, M. A. (2004). Model comparison methods. Methods in Enzymology, 383, 351-366. doi:10.1016/S0076-6879(04)83014-3

Newell, A., \& Rosenbloom, P. S. (1981). Mechanisms of skill acquisition and the law of practice. In J. R. Anderson (Ed.), Cognitive skills and their acquisition (pp. 1-55). Hillsdale, NJ: Erlbaum.

Payne, J. W., Bettman, J. R., \& Johnson, E. J. (1992). Behavioral decision research: A constructive processing perspective. Annual Review of Psychology, 43, 87-131. doi:10.1146/annurev.ps.43.020192.000511

Payne, J. W., Bettman, J. R., \& Johnson, E. J. (1993). The adaptive decision maker. Cambridge, England: Cambridge University Press.

Raftery, A. E. (1995). Bayesian model selection in social research. Sociological Methodology, 25, 111-196. doi:10.2307/271063

Rupinski, M. T., \& Dunlap, W. P. (1996). Approximating Pearson productmoment correlations from Kendall's tau and Spearman's rho. Educational and Psychological Measurement, 56, 419-429. doi:10.1177/ 0013164496056003004

Schmidt, F. L. (1995). Why all banding procedures in personnel selection are logically flawed. Human Performance, 8, 165-177. doi:10.1207/ s15327043hup0803_3

Schwarz, G. E. (1978). Estimating the dimension of a model. Annals of Statistics, 6, 2, 461-464. doi:10.1214/aos/1176344136

Shepard, R. N. (1962). The analysis of proximities: Multidimensional scaling with an unknown distance function. Part I. Psychometrika, 27, 125-140. doi:10.1007/BF02289630

Shepard, R. (1966). Metric structures in ordinal data. Journal of Mathematical Psychology, 3, 287-315.

Simon, H. A. (1956). Rational choice and the structure of the environment. Psychological Review, 63, 2, 129-138. doi:10.1037/h0042769

Steinmann, D. O., \& Doherty, M. E. (1972). A lens model analysis of a bookbag and poker chip experiment: A methodological note. Organizational Behavior\& Human Performance, 8, 450-455. doi:10.1016/00305073(72)90062-1

Tenenbaum, J. B., Griffiths, T. L., \& Kemp, C. (2006). Theory-based Bayesian models of inductive learning and reasoning. Trends in Cognitive Sciences, 10, 309-318. doi:10.1016/j.tics.2006.05.009

Thompson, C. A., \& Opfer, J. E. (2008). Costs and benefits of representational change: Effects of context on age and sex differences in magnitude estimation. Journal of Experimental Child Psychology, 101, 20 51. doi:10.1016/j.jecp.2008.02.003

Trujillo-Ortiz, A., \& Hernandez-Walls, R. (2003). Mskekur: Mardia's multivariate skewness and kurtosis coefficients and its hypotheses testing. A MATLAB file [WWW document]. Retrieved from http://www.mathworks .com/matlabcentral/fileexchange/loadFile.do?objectId $=3519$

Trujillo-Ortiz, A., Hernandez-Walls, R., Barba-Rojo, K., \& Cupul-Magana, L. (2007). HZmvntest: Henze-Zirkler's multivariate normality test. A MAT- 
LAB file [WWW document]. Retrieved from http://www.mathworks.com/ matlabcentral/fileexchange/loadFile.do?objectId $=17931$

Tucker, L. R. (1964). A suggested alternative formulation in the developments by Hursch, Hammond, and Hursch and by Hammond, Hursch, and Todd. Psychological Review, 71, 528-530. doi:10.1037/h0047061

York, K. M., Doherty, M. E., \& Kamouri, J. (1987). The influence of cue unreliability in a multiple cue probability learning task. Organizational Behavior and Human Decision Processes, 39, 303-317. doi:10.1016/ 0749-5978(87)90026-4

Young, F. M., De Leeuw, J., \& Takane, Y. (1976). Regression with qualitative and quantitative variables: An alternating least squares method with optimal scaling features. Psychometrika, 41, 505-528.

\section{Appendix}

\section{Implementation Details for the Least Squares Procedures in the Analysis of Cognitive Ability Data}

The model competition for modeling the Engle et al. (1999) cognitive ability data included GeMM, OLS-BIC, OLS-Wald, and four additional techniques including two versions of robust regression (one using the Huber weighting function and one using the bi-square weighting function), Bayesian regression with normal priors, and ridge regression. Robust regression is a technique used in contexts for reducing the impact of extreme scores and is therefore less sensitive to the presence of outliers. Both robust procedures were implemented within MatLab using a WALD test. The Bayesian and ridge regression procedures were implemented in SAS, with code written by the authors. The Bayesian model used normal priors with variable selection based on the Wald 95\% credible interval. We implemented ridge regression, which is a variant of OLS that applies a shrinkage penalty, lambda, to the predictors. Although this penalty slightly biases the parameter estimates, it can lead to substantial decreases in their variance when the predictors are highly collinear. We employed a variant of the BIC (see Equation A1) to select the lambda for each estimation sample that optimally traded fit (residual sums of squares; RSS) with a measure of flexibility (effective $d f$ ). The parameter estimates associated with the best fit lambda were then applied to the cross-validation sample.

$$
\begin{aligned}
\mathrm{BIC}_{\lambda} & =\log \mathrm{RSS}_{\lambda}+d f_{\lambda} / n \log n, \text { where } d f_{\lambda} \\
& =\operatorname{trace}\left[X\left(X^{t} X+\lambda I\right)^{-1} X^{t}\right]
\end{aligned}
$$

For all procedures, we randomly sampled $50 \%$ of the total sample to form an estimation sample and used the remaining $50 \%$ as the holdout (cross-validation) sample. For each run, we estimated the model on the estimation sample and then applied the statistical model to predict observations in the crossvalidation sample. This was repeated 200 times for each approach. For the sake of comparison, we used the model based on the full sample as the criterion for evaluating statistical power. When the full sample was used, five approaches (OLS-BIC, OLS-Wald, Huber robust least squares, bi-square robust least squares, and Bayesian-Wald) identified a two-parameter model consisting of quantitative SAT and Cattell's culture fair test as the best fit model for predicting Raven's Progressive Matrices. Ridge regression is not typically implemented within a variable selection algorithm, so it was implemented by including all nine predictors. Table 1 presents the fit indices from the crossvalidation exercise. Table 2 presents the probability that each parameter was recovered for $N / 2$.

Received June 3, 2010

Revision received October 20, 2011

Accepted December 19, 2011 